\title{
Dual Virtual Element Methods for Discrete Fracture Matrix models
}

\author{
Alessio Fumagalli*, and Eirik Keilegavlen \\ Department of Mathematics, University of Bergen, 5007 Bergen, Norway
}

Received: 5 December 2017 / Accepted: 6 February 2019

\begin{abstract}
The accurate description of fluid flow and transport in fractured porous media is of paramount importance to capture the macroscopic behavior of an oil reservoir, a geothermal system, or a $\mathrm{CO}_{2}$ sequestration site, to name few applications. The construction of accurate simulation models for flow in fractures is challenging due to the high ratio between a fracture's length and width. In this paper, we present a mixeddimensional Darcy problem which can represent the pressure and Darcy velocity in all the dimensions, i.e. in the rock matrix, in the fractures, and in their intersections. Moreover, we present a mixed-dimensional transport problem which, given the Darcy velocity, describes advection of a passive scalar into the fractured porous media. The approach can handle both conducting and blocking fractures. Our computational grids are created by coarsening of simplex tessellations that conform to the fracture's surfaces. A suitable choice of the discrete approximation of the previous model, by virtual finite element and finite volume methods, allows us to simulate complex problems with a good balance of accuracy and computational cost. We illustrate the performance of our method by comparing to benchmark studies for two-dimensional fractured porous media, as well as a complex three-dimensional fracture geometry.
\end{abstract}

\section{Introduction}

Fractures and faults can strongly influence fluid flow in a porous media, acting, depending on their permeability and porosity, as a preferential path or a barrier. As fracture aperture is several orders of magnitude smaller than any other characteristic size in the domain, fracture modeling is one of the main challenges in subsurface problems.

Geological movements, chemical reaction, or infilling processes may substantially alter the local orientation and composition of the material present in the fractures, leading to anisotropies and strong heterogeneities in both fractures and their intersections. It is thus crucial to be able to include also these phenomenological aspects in the conceptual model.

Applications where fractures can be determinant for reservoir behavior include exploitation of geothermal system, $\mathrm{CO}_{2}$ storage and sequestration, enhanced oil recovery, and nuclear waste disposal. In these fields the solution may dramatically depend on the presence of fractures, thus a correct derivation of suitable mathematical models and their accurate numerical solution is essential.

Our computational model is based on a Discrete Fracture Matrix (DFM) approach, e.g. [1], which aims to

\footnotetext{
* Corresponding author: alessio.fumagalli1984@gmail.com
}

balance computational cost and modeling accuracy in the presence of fractures on multiple scales. Small scale fractures are incorporated in the matrix permeability by analytical or numerical upscaling techniques (e.g. see [2-4] and [5-7]), thus only macroscopic fractures and faults are considered and explicitly described. However, the computational cost associated with their equi-dimensional representation in the grid is still prohibitively high. Following the idea presented, among the others, in references [8-15] fractures and faults are represented as lower-dimensional objects embedded in the rock matrix. This approach is commonly denoted reduced models, hybrid-dimensional models, and mixed-dimensional models. In this approach, fracture aperture becomes a coefficient in the equations, instead of a geometrical constraint for grid generation. The flow equations are averaged along the normal direction of the fractures, yielding a new set of reduced models that are suited for the co-dimensional, that is, lowerdimensional, description of fluid flow. Suitable coupling conditions are necessary to exchange information between all the objects (rock matrix, fractures, and their intersections).

The reduction of dimensionality is an essential step to overcome most of the difficulties associated to the problem. However, in the presence of several fractures that together form a complex network, more advanced numerical 
techniques are crucial to obtain an accurate solution in a reasonable amount of time. The main aspect is the geometrical treatment of the fracture grid with respect to the rock matrix grid. Popular approaches can be classified according to whether the grid in the rock matrix matches the fracture surfaces or not. The matching case can further be split into methods that assume full conformity between the rock matrix and fracture grid, and methods that relaxe this assumption.

In the class of conforming methods, where fracture grids are composed by faces of the rock matrix grid, several numerical schemes have been considered, including finite elements [13, 16-19], finite volumes [6, 20-23], Mimetic Finite Differences (MFD) [24, 25], and the newly introduced Virtual Finite Elements (VEM) [26-28]. For the latter see also [29-33] for the non-fractured case. All these methods highlight specific advantages for example related to local mass conservation, capability to be implemented in standard software packages, or relax some constraints on grid cell shapes to name a few.

In the class of non-conforming discretization, the main tool is a mortar coupling between the rock matrix grid and the fracture grids. The rock mesh is constrained with the position of the fractures but not strictly with the actual fracture meshes, and vice versa. Some examples are reported in $[10,27,34]$ where different type of mortar variables as well as numerical schemes are considered. The mortar variables allow for a generalized formulation that unifies conforming and non-conforming approaches [35].

Finally, a fully non-matching coupling among the grids requires ad-hoc solutions to establish a communication between the fractures and the rock matrix. One possibility is the class of eXtended Finite Element Methods (XFEM) [36-41], where a local enrichment with new basis functions is considered to handle the non-conformity, or the class of Embedded Discrete Fracture Matrix Methods (EDFM) [5, 41-43], where approximate formulas for fracture-matrix transmissibilities are computed based on geometrical considerations.

In this paper we consider a conforming discretization with the dual virtual element approximation to simulate a mixed-dimensional Darcy problem and a finite volume discretization for the mixed-dimensional transport problem. The first choice is motivated by the flexibility of the VEMs with respect to the shape of the grid cells. With this choice it is possible to relax most of the difficulties related with conforming discretization, e.g. the computational cost associated to resolve, by the rock matrix grid, a complex system composed of several intersecting fractures. Moreover, this approximation ensures local mass conservation and is able to consider heterogeneous and anisotropic permeability tensors. The Darcy velocity can thus be used in the mixeddimensional transport problem. In this case we consider an upwind scheme, extended to handle the mixed-dimensional nature of the problem.

The paper is structured as follows: In Section 2, we present both the physical equations and the reduced model, with the interface conditions that couple the matrixfracture system and the fracture-fracture system for both the Darcy and transport problems. Section 3 deals with the weak and integral formulation of the previous physical processes. In Section 4, we present the numerical discretization of the problem with a highlight on the enrichment of the finite element spaces. In Section 5, we present some numerical experiments to assess the effectiveness of the proposed method. Finally, Section 6 is devoted to conclusion and to ongoing work.

\section{Mathematical model}

In this section, we introduce the mathematical models in the mixed-dimensional setting, i.e. equations that couple different spatial dimensions and together describe the quantities of interest. We present two mathematical models useful for subsurface simulations: the mixed-dimensional Darcy problem, presented in Section 2.1, to describe the flow and pressure, and the mixed-dimensional transport problem, presented in Section 2.2, to describe the motion of a passive scalar transported by the Darcy velocity.

The motivation for the mixed-dimensional formulation is the observation that the fracture aperture is orders of magnitude smaller than other characteristic sizes of the problem. Hence, a straightforward mesh construction with the two fracture surfaces as constraints would produce high numbers of cells and/or low-quality cells due to high aspect ratios or sharp angles. To avoid this geometrical constraint, fractures are represented as lower dimensional objects embedded in the rock matrix. Fracture intersections, and their intersections again, are considered as objects of even lower dimensions. To be specific, in a three-dimensional domain, we consider fracture surfaces as $2 \mathrm{~d}$ objects, the intersection between two (or more) fractures form a $1 \mathrm{~d}$ line, and two (or more) intersection lines can meet in a 0d point. The physical processes are described via reduced models with suitable coupling conditions among the objects of different dimensions. The fracture aperture is now part of the equations and not any more a geometrical constraint. For more details on the derivation of the reduced model we refer to [8-11, 13, 26-28, 37, 39, 40, 44, 45].

In the reduced model, it is a common choice to consider the reduced scalar variables as averaged and the vector variables as integrated along cross section of the fracture. In this work we follow this approach.

In the sequel we indicate by $(\cdot, \cdot)_{A}$ the scalar product in $L^{2}(A)$. Moreover, the trace operator on a domain $A$ will be indicated by $\left.\cdot\right|_{A}$.

\subsection{Mixed-dimensional Darcy problem}

Let us consider a regular domain $\Omega \subset \mathbb{R}^{N}$, for $N>0$, with outer boundary $\partial \boldsymbol{\Omega}$. The domain $\Omega$ is composed by a single, possibly non-connected, equi-dimensional domain $\Omega^{N}$ and several lower-dimensional domains $\Omega^{d}$ for $d<N$, which are possibly non-connected. Clearly $\cup_{d=0, \ldots, N} \Omega^{d}=\Omega$ and $\Omega^{d} \cap \Omega^{d^{\prime}}=\emptyset$ for $d \neq d^{\prime}$. However, we indicate with $\Gamma^{d}=\partial \boldsymbol{\Omega}^{d} \cap \boldsymbol{\Omega}^{d-1}$, which is geometrically equivalent to $\Omega^{d-1}$ but it will be more convenient to keep them separate. We have $\Gamma=\cup_{d=0, \ldots, N} \Gamma^{d}$. See Figure 1 as an example of the subdivision. 


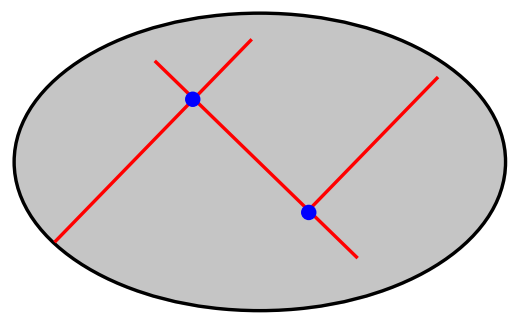

Fig. 1. Domain subdivision for $N=2$. In gray $\Omega^{2}$, in red $\Omega^{1}$, and in blue $\Omega^{0}$.

We are interested in the mixed-dimensional Darcy problem which describes the pressure and velocity fields in $\Omega$. Following the idea presented in [10], we define the pressure compound $p=\left(p^{0}, \ldots, p^{N}\right)$ which represents the pressure in each dimension, indicated as superscript. Similarly, we introduce the velocity compound $\boldsymbol{u}=\left(0, \boldsymbol{u}^{1}, \ldots, \boldsymbol{u}^{\hat{N}}, 0\right)$, which is the velocity field defined in $\Omega^{N}$ or in the tangential spaces of each dimension $d<N$. The meaning of the two $0 \mathrm{~s}$ will be clarified below. In the following problem, each dimension is coupled with the lower dimension; we consider thus two normals $\boldsymbol{n}$ associated to a $d<N$-dimensional manifold with respect to its "two sides", indicated by $\boldsymbol{n}_{+}$and $\boldsymbol{n}_{-}$. Each disconnected object with dimension $d<N$ when immersed in the surrounding $d+1$-manifold, it is assumed to be an orientable $d$-dimensional manifold, with unique normal $\boldsymbol{n}$ lying on the tangent space of the $d+1$-manifold. This implies $\boldsymbol{n}_{+}=-\boldsymbol{n}_{-}$, an illustration is given in Figure 2 . In the particular case of $T$-type intersection only one side can be defined and the following equations should be understood in this sense. Given a side, by convention the normal vector points from the higher dimensional to the lowerdimensional domain. Moreover, we indicate by the same subscript $\cdot \cdot_{ \pm}$the restriction of elements in $\Omega^{d}$ defined on each side of $\Gamma^{d}$ through a suitable trace operator.

\subsubsection{Fixed-dimensional Darcy problem}

We consider the Darcy problem for the pressure and velocity as

$$
\begin{gathered}
\boldsymbol{u}+K \nabla p=\mathbf{0} \\
\nabla \cdot \check{\boldsymbol{u}}-\llbracket \hat{\boldsymbol{u}} \cdot \hat{\boldsymbol{n}} \rrbracket=\check{f}
\end{gathered}
$$

The $\cdot$ notation means "on the higher dimensional object"

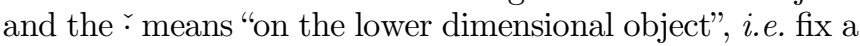
dimension $d$ the second of (1a) becomes

$$
\nabla \cdot \boldsymbol{u}^{d}-\llbracket \boldsymbol{u}^{d+1} \cdot \boldsymbol{n}^{d+1} \rrbracket=f^{d} \quad 0 \leq d \leq N .
$$

In the system (1a) the first equation and second equations are defined on domains of the same dimension. For lower dimensional objects, the differential operators are defined in the tangent plane of the objects; in the special 0d case these objects are void. $K$ represents the effective permeability compound $K=\left(1, K^{1}, \ldots, K^{N}\right)$, which is a collection of symmetric and positive defined tensors. For $d<N, K^{d}$ is interpreted as laying in the tangent plane of the relevant physical object. Model (1a) allows for permeability variations both (a)

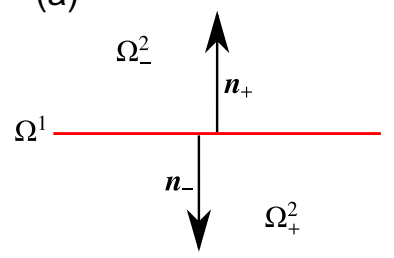

(b)

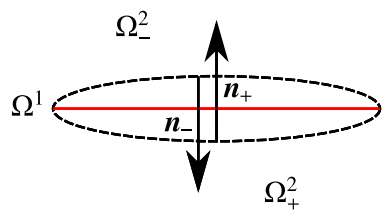

(c)

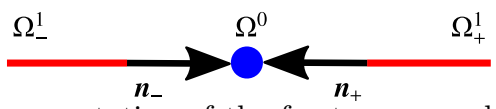

Fig. 2. Representation of the fracture normal associated with respect to each "side" ( + and - ). (a) A bidimensional domain $\Omega^{2}$, (b) The fracture is fictitiously enlarged. (c) For a 0d intersection.

between all objects considered, and spatially within each object. The permeabilities are scaled by the "aperture" of each dimension: Denote by $\epsilon^{d}$ the length $(N-1)$, area $(N-2)$, or cross-sectional volume $(N-3)$. The permeability of a lower $d$-dimensional $(d<N)$ object is then defined as $K^{d}=\epsilon^{d} \widetilde{K}^{d}$, with $\widetilde{K}^{d}$ the permeability of its equi-dimensional representation. The unit of measure of $K^{d}$ is thus $\left[\mathrm{m}^{4-d} / \mathrm{s}\right]$. The source or sink term $f$ is defined as the compound $f=\left(f^{0}, \ldots, f^{N}\right)$, which are scalar functions defined in each dimension. Finally, the jump operator $\llbracket \hat{\boldsymbol{u}} \cdot \hat{\boldsymbol{n}} \rrbracket=\left.\sum_{+} \hat{\boldsymbol{u}} \cdot \hat{\boldsymbol{n}}\right|_{+}$is imposed on each side of $\boldsymbol{\Omega}^{d-1}$ viewed as immersed in $\Omega^{d}$.

The first equation in (1a) is the generalized Darcy law in each dimension, note that for the 0d case it is simply an identity. The second equation of (1a) represents conservation of mass: The divergence term prescribes conservation of mass within the dimension, while the jump term represents the inflow/outflow from the higher dimension. Interaction with the lower dimension is incorporated into the boundary conditions, see Section 2.1.3. Due to the definition of the velocity compound, for $d=N$ equation (1a) the inflow/outflow from the "higher dimension" is null. For $d=0$ the conservation of mass equation represents the continuity of the fluxes for the 1-dimensional objects involved in the intersection.

We emphasize that the mixed-dimensional problem only considers direct interaction between objects one dimension apart. That is, quantities defined on $\Omega^{d-1}$ are in communication with the quantities defined on $\Omega^{d}$, but not directly with quantities defined on $\Omega^{d+1}$.

\subsubsection{Flow between dimensions}

The coupling conditions between two subsequent dimensions are defined for each side as

$$
\left.\hat{\boldsymbol{u}} \cdot \hat{\boldsymbol{n}}\right|_{ \pm}+\hat{k}_{*}\left(\check{p}-\left.\hat{p}\right|_{ \pm}\right)=0 \quad \text { on } \Gamma .
$$

The $k_{*}$ is the effective normal permeability, defined as $k_{*}=\left(k_{*}^{0}, \ldots, k_{*}^{N-1}\right)$. Clearly, equation (1b) is valid between dimension $d$ and $d+1$ for $0 \leq d<N$. Note that in equation (1b) it is possible to define different effective normal permeability on each side \pm , however, to simplify the notation, we assume only one single value. 


\subsubsection{Boundary conditions}

To obtain a well-posed problem, boundary conditions must be assigned on the external boundary $\partial \Omega$ and the boundary of the sub-domains of each dimension, $\Omega^{d}$. We indicate by $\partial \Omega_{\text {out }}^{d}$ the (possibly empty) portion of the boundary of $\Omega^{d}$ that intersects the boundary of $\Omega$, for $d>0$. We introduce also $\partial \Omega_{\text {in }}^{d}$ the portion of boundary of $\Omega^{d}$ that does not intersect with $\partial \Omega$, for $d>0$. $\partial \Omega_{\text {in }}^{d}$ is further split into the internal boundaries $\Gamma^{d}$, which are formed by the embedding of objects in $\Omega^{d-1}$ into $\Omega^{d}$, and the remaining part, $\partial \Omega_{\text {in }}^{d} \backslash \Gamma^{d}$.

For simplicity we assume only pressure boundary conditions on $\partial \Omega_{\text {out }}^{d}$, given as

$$
p^{d}=\bar{p}^{d} \quad \text { on } \partial \Omega_{\text {out }}^{d},
$$

with $\bar{p}^{d}$ is a given pressure at the boundary for each $d>0$. On the internal boundary $\Gamma^{d}$, we impose flux conditions that represent the inflow and outflow from lower dimensions, in accordance with (1b). In practice, this couples the flux between dimensions with the pressure in both dimensions, in what can be interpreted as a Robin-type condition.

On the remaining internal portion $\partial \Omega_{\text {in }}^{d} \backslash \Gamma^{d}$, for $d>0$, we assign the so-called "tip-condition", namely

$$
\left.\boldsymbol{u}^{d} \cdot \boldsymbol{n}\right|_{\partial \Omega_{\mathrm{in}}^{d}}=0 \quad \text { on } \partial \boldsymbol{\Omega}_{\mathrm{in}}^{d},
$$

where in the previous equation $\boldsymbol{n}$ stands for the outward unit normal of $\partial \boldsymbol{\Omega}_{\text {in }}^{d}$.

\subsubsection{Generalized formulation}

Equation (1) is the mixed-dimensional Darcy problem, formulated in terms of pressure and velocity. The problem (1) may be recast into a pure pressure formulation, however the numerical scheme introduced in Section 4 considers explicitly the pressure and velocity fields as unknowns.

Following the idea presented in $[10,34,46]$, we can write problem (1) in a more compact form similar to the standard Darcy formulation. To this end, we introduce the following divergence operator $\mathfrak{D}$. between dimensions as such that

$$
\mathfrak{D} \cdot \boldsymbol{w}=\nabla \cdot \check{\boldsymbol{w}}-\llbracket \hat{\boldsymbol{w}} \cdot \hat{\boldsymbol{n}} \rrbracket,
$$

as well as a mixed-dimensional gradient operator $\mathfrak{D}$ such that

$$
\mathfrak{D} q=[\nabla \check{q}, \check{q}-\hat{q}]^{T} .
$$

Considering the Darcy velocity composed by $\boldsymbol{u}$ in $\Omega$ and $\boldsymbol{u} \cdot \boldsymbol{n}$ on $\boldsymbol{\Gamma}$, system (1) becomes

$$
\begin{array}{ll}
\boldsymbol{u}+\mathfrak{S D} p=0 & \text { in } \Omega \times \Gamma \\
\mathfrak{D} \cdot \boldsymbol{u}=f & \text { in } \Omega
\end{array}
$$

In the previous equation $\mathfrak{i}$ is defined accordingly to include both the effective tangential and normal permeabilities.

\subsection{Mixed-dimensional transport problem}

Once the problem (1) is solved, the velocity field $\boldsymbol{u}$ can be used to describe the mixed-dimensional transport problem. We consider the same splitting of $\Omega$ as in Section 2.1.
We introduce the concentration compound as $c=$ $\left(0, c^{1}, \ldots, c^{N}, 0\right)$, with $0 \leq c \leq 1$ for all $d$, to describe the portion of a passive tracer in a grid cell. We indicate by $t$ the time variable and $(0, T)$ the time interval. The proposed model extends to multiple fracture networks the model presented in [47] with multi-dimensional treatment of fracture intersections. Other important references on this subject are $[20,22]$.

\subsubsection{Fixed-dimensional transport problem}

The model is the following, given $\boldsymbol{u}$ find $c$ such that

$$
\check{\phi} \check{\epsilon} \partial_{t} \check{c}+\nabla \cdot(\check{\boldsymbol{u}} \check{c})-\llbracket \hat{\boldsymbol{u}} \cdot \hat{\boldsymbol{n}} \hat{\boldsymbol{c}} \rrbracket=\check{r} \quad \text { in } \Omega \times(0, T),
$$

where we have indicated with $\partial_{t} c$ the time derivative of $c$, while $\phi$ and $r$ are the porosity and a scalar source/sink term in each dimension, represented as compounds. Finally, $\epsilon$ represents the "fracture aperture" in each dimension, we have $\epsilon=\left(\epsilon^{0}, \ldots, \epsilon^{N-1}, 1\right)$. We assume that $\epsilon^{d}>0$ for all $0 \leq d \leq N$. For simplicity we assume that $\epsilon$ does not depend on time.

In (2a), the divergence term models the conservation of $c$ in the current dimension $d$ and the flow exchange with the lower dimension $d-1$. The jump operator describes the flow exchange with the higher dimension $d+1$. The coupling conditions, related to the definition of inflow/outflow of the lower-dimensional objects, associated to (2a) are

$$
\left.\hat{c}\right|_{ \pm}(\boldsymbol{x})=\left\{\begin{array}{ll}
\left.\hat{c}\right|_{ \pm}(\boldsymbol{x}) & \text { if }\left.\hat{\boldsymbol{u}} \cdot \hat{\boldsymbol{n}}\right|_{ \pm} \geq 0 \\
\check{c}(\boldsymbol{x}) & \text { otherwise }
\end{array} \text { on } \Gamma \times(0, T) .\right.
$$

Also in this case, problem (2) couples the concentration defined on $\Omega^{d}$ with $\Omega^{d-1}$ and $\Omega^{d+1}$, but not directly with $\Omega^{d-2}$ or $\Omega^{d+2}$.

\subsubsection{Initial and boundary conditions}

With $(2 a)$ we need to assign initial condition $\overline{\bar{c}}$ for the concentration $c$, as

$$
c\left(t=t_{\text {ini }}\right)=\overline{\bar{c}} \text { on } \Omega \times\{0\},
$$

with $t_{\text {ini }}$ the initial time. Finally, on the inflow part of the outer boundary we assign a boundary condition $\bar{c}$ for (2a)

$$
c=\bar{c} \quad \text { on } \partial \Omega_{\mathrm{out}, \boldsymbol{u} \cdot \boldsymbol{n}>0}^{d} \times(0, T),
$$

where $\partial \boldsymbol{\Omega}_{\text {out } \boldsymbol{u} \cdot \boldsymbol{n}>0}^{d}$ is the portion of $\partial \boldsymbol{\Omega}_{\text {out }}^{d}$ such that an inflow occurs. Equation (2) is the mixed-dimensional transport problem.

\subsubsection{Generalized formulation}

The problem (2) can be condensed, using the formalism introduce at the end of the previous subsection, as

$$
\phi \epsilon \partial_{t} c+\mathcal{D} \cdot(\boldsymbol{u c})=r \quad \text { in } \Omega \times(0, T),
$$

which is a general form of a conservative equation in the mixed-dimensional setting. 


\section{Weak and integral formulation}

In the view of introducing the numerical approximations, in this part we consider the weak formulation of (1) and the integral formulation of (2).

\subsection{Weak formulation of mixed-dimensional Darcy problem}

We introduce the functional spaces suitable to approximate the pressure and the velocity. The domains $\Omega^{d}$ may contain cuts or internal interfaces, in this case refer to [17, 34, 48] for a more detailed description of functional setting. Considering a fixed-dimensional domain $\Omega^{d}$ we have for the pressure $p^{d} \in Q^{d}=L^{2}\left(\Omega^{d}\right)$, for $d>0$, and $p^{0} \in Q^{0}=\mathbb{R}$. For the velocity we consider, for $d>0$, the Hilbert space:

$$
V^{d}=\left\{\boldsymbol{v} \in H_{\mathrm{div}}\left(\Omega^{d}\right):\left.\boldsymbol{v} \cdot \boldsymbol{n}\right|_{ \pm} \in L^{2}\left(\Gamma^{d-1}\right)\right\} .
$$

The additional condition in $V^{d}$ is related to the Robin-type nature of the condition (1b), see [13, 28]. The global spaces $Q$ and $V$ are defined as the union of the local spaces.

We introduce now the bilinear forms associated to the problem (1). We have $a^{d}(\cdot, \cdot): V^{d} \times V^{d} \rightarrow \mathbb{R}$ and $b^{d}(\cdot, \cdot): V^{d} \times Q^{d} \rightarrow \mathbb{R}$, defined as

$$
a^{d}(\boldsymbol{w}, \boldsymbol{v})=\left(K^{-1} \boldsymbol{w}, \boldsymbol{v}\right)_{\Omega^{d}} \quad b^{d}(\boldsymbol{w}, q)=(\nabla \cdot \boldsymbol{w}, q)_{\Omega^{d}},
$$

with $\boldsymbol{w}, \boldsymbol{v} \in V^{d}$ and $q \in Q^{d}$. For the coupling between dimensions, $d>0$, we introduce $\alpha^{d}(\cdot, \cdot): V^{d} \times V^{d} \rightarrow \mathbb{R}$ and $\beta^{d, d-1}(\cdot, \cdot): V^{d} \times Q^{d-1} \rightarrow \mathbb{R}$ defined as

$$
\begin{gathered}
\alpha^{d}(\boldsymbol{w}, \boldsymbol{v})=\sum_{ \pm}\left(\left.\eta_{*}^{d} \boldsymbol{w} \cdot \boldsymbol{n}\right|_{ \pm},\left.\boldsymbol{v} \cdot \boldsymbol{n}\right|_{ \pm}\right)_{\Omega^{d-1}}, \\
\beta^{d, d-1}(\boldsymbol{w}, q)=\sum_{ \pm}\left(\left.\boldsymbol{w} \cdot \boldsymbol{n}\right|_{ \pm}, q\right)_{\Omega^{d-1}},
\end{gathered}
$$

where $\boldsymbol{w}, \boldsymbol{v} \in V^{d}$ and $q \in Q^{d-1}$. In the previous definition we have $\eta_{*}^{d}$ the inverse of $k_{*}^{d}$ and $\boldsymbol{n}$ the normal of $\Omega^{d-1}$ in the tangent space of $\Omega^{d}$. The global bilinear forms are defined by $a(\cdot, \cdot): V \times V \rightarrow \mathbb{R}$ and by $b(\cdot, \cdot): V \times Q \rightarrow \mathbb{R}$ as

$$
\begin{gathered}
a(\boldsymbol{w}, \boldsymbol{v})=\sum_{d>0} a^{d}\left(\boldsymbol{w}^{d}, \boldsymbol{v}^{d}\right)+\alpha^{d}\left(\boldsymbol{w}^{d}, \boldsymbol{v}^{d}\right), \\
b(\boldsymbol{w}, q)=\sum_{d>0} b^{d}\left(\boldsymbol{w}^{d}, q^{d}\right)+\beta^{d, d-1}\left(\boldsymbol{w}^{d}, q^{d-1}\right),
\end{gathered}
$$

with $\boldsymbol{w}, \boldsymbol{v} \in V$ and $q \in Q$. We can introduce the weak formulation of (1), which reads: find $(\boldsymbol{u}, p) \in V \times Q$ such that

$$
\begin{array}{ll}
a(\boldsymbol{u}, \boldsymbol{v})+b(\boldsymbol{v}, p)=G(\boldsymbol{v}) & \forall \boldsymbol{v} \in V \\
b(\boldsymbol{u}, q)=F(q) & \forall q \in Q
\end{array}
$$

where the functionals are defined as

$$
F(q)=\sum_{d}\left(f^{d}, q^{d}\right)_{\Omega^{d}} \quad G(\boldsymbol{v})=-\sum_{d>0}\left(\left.\boldsymbol{v} \cdot \boldsymbol{n}\right|_{\partial \Omega_{\text {out }}^{d}}, \bar{p}^{d}\right)_{\partial \Omega_{\text {out }}^{d}} .
$$

\subsection{Integral formulation of mixed-dimensional transport problem}

In Section 4.2 we consider a finite volume approximation of problem (2), thus we introduce here its integral formulation.

Considering a suitable sub-domain of $\Omega^{d}$ called $E$, later will be a cell of the computational grid, we integrate equation (2) obtaining the following

$$
\begin{aligned}
& \left(\check{\phi} \check{\epsilon} \partial_{t} \check{c}, 1\right)_{E}+\left(\left.\check{\boldsymbol{u}} \cdot \check{\boldsymbol{n}}\right|_{\partial E},\left.\check{c}\right|_{\partial E}\right)_{\partial E} \\
& +\sum_{ \pm}\left(\left.\check{\boldsymbol{u}} \cdot \check{\boldsymbol{n}}\right|_{I, \pm},\left.\check{c}\right|_{I, \pm}\right)_{I, \pm}-\left(\left.\hat{\boldsymbol{u}} \cdot \hat{\boldsymbol{n}}\right|_{E, \pm},\left.\hat{c}\right|_{E, \pm}\right)_{E}=(\check{r}, 1)_{E},
\end{aligned}
$$

where $I=E \cap \Omega^{d-1}$ is the intersection between $E$ and the lower dimensional domain $\Omega^{d-1}$, which can be an empty set.

In (4), we notice the term depending on $I$ which describes the inflow/outflow exchange between the current and lower dimensions. Condition (2b) applies also in this case.

\section{Numerical discretization}

In this section we introduce the numerical scheme used to solve the mixed-dimensional problems (1) and (2). In particular, for the former we consider the lowest order dual VEM approximation, while for the latter we employ a finite volume discretization with an upwind scheme and implicit Euler in time.

Let us introduce a suitable tessellation $\mathcal{T}$ of $\Omega$ in polytopes indicated by $E$, such that

$$
\mathcal{T}=\cup_{i} E_{i} \quad \text { and } \quad E_{i} \cap E_{j}=\emptyset \text { for } i \neq j .
$$

We denote by $h_{E}$ the diameter of $E$, by $\boldsymbol{x}_{E}$ the center of $E$, by $\mathcal{E}(E)=\{e \in \partial E\}$ the set of faces for the cell $E$, and by $\boldsymbol{n}_{e}$ with $e \in \mathcal{E}(E)$ the unit normal of $e$ pointing outward with respect to the internal part of $E$. The term faces indicates proper faces for the $3 \mathrm{~d}$ grid, edges for the $2 \mathrm{~d}$ grids, and vertexes for the $1 \mathrm{~d}$ grids. We indicate also by $h=\max _{E \in \mathcal{T}} h_{E}$ the characteristic grid size and by $\mathcal{E}=\cup_{E \in \mathcal{T}} \mathcal{E}(E)$ the set of grid faces. It is important to note that the polytopes may have different spatial dimension, i.e. they are $3 \mathrm{~d}$ or $2 \mathrm{~d}$ or $1 \mathrm{~d}$ or $0 \mathrm{~d}$ objects. However, fixing a single dimension $d$ the cells of the grid belongs to $\Omega^{d}$.

For simplicity, both problems (1) and (2) use the same computational grid.

In Section 4.1 we present the discretization for problem (1), while in Section 4.2 the numerical approximation for problem (2). Finally, in Section 4.3 we introduce a coarsening strategy used in the numerical examples.

\subsection{Mixed-dimensional dual VEM discretization}

We present a numerical scheme to solve the mixed-dimensional Darcy problem (1) in presence of polytopes in the grid. The resulting scheme will be locally and globally conservative, thus suitable to approximate the velocity field used in (2). We consider the dual VEM of lowest order 
degree, for more details on the derivation and on the analysis of the method, see [26-33].

\subsubsection{Discrete function spaces}

We introduce finite dimensional spaces to approximate the Darcy velocity $\boldsymbol{u}$ and the pressure $p$ in each element of the grid. For simplicity we consider a single dimension and, if not essential, we drop the superscript $d$ to simplify notation. Again, the differential operators are understood to be defined on the tangential space. Given an element $E$ the local discretization space for the pressure is $Q_{h}^{d}(E)=$ $\mathbb{P}_{0}(E) \subset L^{2}(E)$, where $\mathbb{P}_{r}(E)$ is the space of polynomial of order $r$ on the domain $E$. While higher-order dual VEM approximations are possible, see [31,32], the solution will often have low regularity caused by fractures or general permeability heterogeneities. We therefore stay with the lowest order formulation, as is common practice in the field. For the velocity we need to introduce the following space:

$$
\begin{gathered}
V_{h}^{d}(E)=\left\{\boldsymbol{v} \in H_{\mathrm{div}}(E): \boldsymbol{v} \cdot \boldsymbol{n}_{e} \in \mathbb{P}_{0}(e) \forall e \in \mathcal{E}(E),\right. \\
\left.\nabla \cdot \boldsymbol{v} \in \mathbb{P}_{0}(E), \nabla \times \boldsymbol{v}=\mathbf{0}\right\} .
\end{gathered}
$$

The shape of the functions in $V_{h}^{d}(E)$ is not defined a-priori and is implicitly defined by $V_{h}^{d}(E)$. The curl-free condition is necessary to uniquely define the elements in $V_{h}^{d}(E)$. We indicate by $V_{h}^{d}$ the velocity approximation space in the same dimensional grid and by $V_{h}$ the global discretization space for the velocity formed by the compound $\left(V_{h}^{1}, \ldots, V_{h}^{N}\right)$. Similarly, $Q_{h}^{d}$ indicates the pressure approximation space in the same dimension $d$ while $Q_{h}$ is the global discretization space. For the velocity, we impose to the faces which are not in contact with different dimensions to be single value. Otherwise, the degree of freedom is doubled and connected thorough the coupling condition (1b) to the lower dimensional object. See Figure 3 for an example.

\subsubsection{Approximation of bilinear forms}

With the previous definition of $Q_{h}$ and $V_{h}$ it is immediate to approximate the bilinear form $b$ for all the dimensions as well as the bilinear forms associated with the coupling conditions, $\alpha^{d}$ and $\beta^{d, d-1}$. The functionals $F$ and $G$ are discretized similarly. The bilinear forms $a^{d}$ are not immediately computable with the degrees of freedom introduced, but require the definition of a suitable projection operator. First, we introduce the local space

$$
\mathcal{V}_{h}^{d}(E)=\left\{\boldsymbol{v} \in V_{h}^{d}(E): \boldsymbol{v}=K \nabla v, \text { for a } v \in \mathbb{P}_{1}(E)\right\},
$$

and the projection operator is defined as $\Pi_{0}: V_{h}^{d}(E) \rightarrow \mathcal{V}_{h}^{d}(E)$ such that given $\boldsymbol{v} \in V_{h}^{d}(E)$ we have $a^{d}\left(\boldsymbol{v}-\Pi_{0} \boldsymbol{v}, \boldsymbol{w}\right)=0$ for all $\boldsymbol{w} \in \mathcal{V}_{h}^{d}(E)$. Note that the space $\mathbb{P}_{1}(E)$ in the definition of $\mathcal{V}_{h}^{d}(E)$ will be approximated by a (tangential) monomial basis. Where we are assuming a constant value of the permeability in the element $E$. For (5) to make sense, the permeability must be constant in each cell. As discussed above, the permeability will in practice often be spatially varying on all scales due to the presence of smaller scale heterogeneities. Inclusion of spatially varying permeabilities within a computational

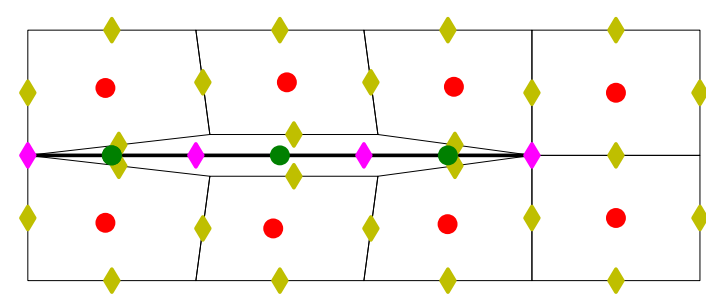

Fig. 3. Representation of the degrees of freedom for a $2 \mathrm{~d}$ and $1 \mathrm{~d}$ grid. The pressure dof are represented by circles, red for the $2 \mathrm{~d}$ grid and green for the $1 \mathrm{~d}$. The velocity dof are depicted by yellow diamonds for the $2 \mathrm{~d}$ grid and purple diamonds for the $1 \mathrm{~d}$. The nodes of the $2 \mathrm{~d}$ grid are moved only for visualization purpose.

cell would require corresponding modifications of the approximation order for pressure and flux. Our preferred approach to including more permeability variations is to stay with lowest order approximations, but instead refine the grid.

With the orthogonality property it is possible to split the bilinear form $a^{d}$ in a term on $\mathcal{V}_{h}^{d}(E)$ and one on the $a^{d}$-orthogonal space of $\mathcal{V}_{h}^{d}(E)$, namely

$$
\begin{aligned}
a^{d}(\boldsymbol{u}, \boldsymbol{v}) & =a^{d}\left(\Pi_{0} \boldsymbol{u}, \Pi_{0} \boldsymbol{v}\right)+a^{d}\left(T_{0} \boldsymbol{u}, T_{0} \boldsymbol{v}\right) \\
& =(K \nabla \boldsymbol{u}, \nabla \boldsymbol{v})_{\Omega^{d}}+a^{d}\left(T_{0} \boldsymbol{u}, T_{0} \boldsymbol{v}\right)
\end{aligned}
$$

with $T_{0}=I-\Pi_{0}$. The first bilinear form is now fully computable with the velocity degrees of freedom introduced before and represents a consistency term with respect to $a^{d}(\boldsymbol{u}, \boldsymbol{v})$. The second term is not yet computable and can be replaced by a stabilization term. Following the ideas presented in [31-33], we approximate the term by,

$$
a^{d}\left(T_{0} \boldsymbol{u}, T_{0} \boldsymbol{v}\right) \approx \varsigma s^{d}\left(T_{0} \boldsymbol{u}, T_{0} \boldsymbol{v}\right),
$$

where $s^{d}: V_{h}^{d}(E) \times V_{h}^{d}(E) \rightarrow \mathbb{R}$ is the bilinear form associated with the stabilization and $\varsigma=\varsigma(d) \in \mathbb{R}^{+}$is a scaling parameter described in the sequel. In details, denoting by $\boldsymbol{\varphi}$ an element of the basis for $V_{h}^{d}(E)$, in our case we consider

$$
s^{d}\left(T_{0} \boldsymbol{\varphi}_{\omega}, T_{0} \boldsymbol{\varphi}_{\theta}\right)=\sum_{i=1}^{N_{\text {dof }}} \operatorname{dof}_{i}\left(T_{0} \boldsymbol{\varphi}_{\omega}\right) \operatorname{dof}_{i}\left(T_{0} \boldsymbol{\varphi}_{\theta}\right),
$$

with $N_{\text {dof }}$ the total number of velocity degrees of freedom of the element $E$ and $\operatorname{dof}_{i}: V_{h}^{d}(E) \rightarrow \mathbb{R}$ is defined as

$$
\operatorname{dof}_{i}\left(\boldsymbol{\varphi}_{\omega}\right)=i \text { th degree of freedom of } \boldsymbol{\varphi}_{\omega}=\delta_{i \omega} .
$$

We introduce the discrete version $a_{h}^{d}: V_{h}^{d}(E) \times V_{h}^{d}(E) \rightarrow \mathbb{R}$ of the bilinear form $a^{d}$, defined as

$$
a_{h}^{d}(\boldsymbol{u}, \boldsymbol{v})=a^{d}\left(\Pi_{0} \boldsymbol{u}, \Pi_{0} \boldsymbol{v}\right)+\varsigma s^{d}\left(T_{0} \boldsymbol{u}, T_{0} \boldsymbol{v}\right),
$$

and the discrete version of the weak problem (3), which reads: find $(\boldsymbol{u}, p) \in V_{h} \times Q_{h}$ such that

$$
\begin{array}{ll}
a_{h}(\boldsymbol{u}, \boldsymbol{v})+b(\boldsymbol{v}, p)=G(\boldsymbol{v}) & \forall \boldsymbol{v} \in V_{h}, \\
b(\boldsymbol{u}, q)=F(q) & \forall q \in Q_{h},
\end{array}
$$


where $a_{h}(\boldsymbol{u}, \boldsymbol{v})=\sum_{d>0} a_{h}^{d}\left(\boldsymbol{u}^{d}, \boldsymbol{v}^{d}\right)+\alpha^{d}\left(\boldsymbol{w}^{d}, \boldsymbol{v}^{d}\right)$. The construction of the discrete problem in term of local matrix computations is described in $[28,30]$.

The stabilization parameter $\varsigma$ is used to impose the scaling on $h$ of the stabilization term equivalent to the consistency term. In practice we require that, for a fixed dimension $d$, there exists $l_{*}, l^{*} \in \mathbb{R}^{+}$, independent from the discretization, such that

$$
\imath_{*} a^{d}(\boldsymbol{v}, \boldsymbol{v}) \leq \varsigma s^{d}(\boldsymbol{v}, \boldsymbol{v}) \leq \imath^{*} a^{d}(\boldsymbol{v}, \boldsymbol{v}) \quad \forall \boldsymbol{v} \in V_{h}^{d} .
$$

Following [28], by a scaling computation we evaluate the dependency of $\varsigma$ from the local grid size $h_{E}$ and obtain the following relation for a fix dimension $d$

$$
\varsigma(E)=h_{E}^{2-d} \text {. }
$$

Note that this expression is local and independent from the maximal dimension $N$ of the problem.

\subsubsection{Matrix formulation}

We introduce the matrix formulation associated with the problem (6). Considering the following matrices:

$$
\begin{gathered}
{\left[A_{d}\right]_{i j}=a_{h}^{d}\left(\boldsymbol{\varphi}_{j}, \boldsymbol{\varphi}_{i}\right)+\alpha^{d}\left(\boldsymbol{\varphi}_{j}, \boldsymbol{\varphi}_{i}\right),} \\
{\left[B_{d}\right]_{i j}=b^{d}\left(\boldsymbol{\varphi}_{j}, \psi_{i}\right), \quad\left[C_{d, d-1}\right]_{i j}=\beta^{d, d-1}\left(\boldsymbol{\varphi}_{j}, \psi_{i}\right),}
\end{gathered}
$$

and vectors

$$
\left[G_{d}\right]_{i}=G\left(\varphi_{i}\right), \quad\left[F_{d}\right]_{i}=F\left(\psi_{i}\right)
$$

where $\phi$ are basis for the pressure and $[\cdot]_{i, j}$ indicates the element $(i, j)$ in the matrix, a similar notation is used for vectors. The global problem reads for $N=3$, solve the following linear system

$$
\left[\begin{array}{cccccccc}
A_{3} & B_{3} & 0 & C_{3,2} & 0 & 0 & 0 & 0 \\
B_{3}^{T} & 0 & 0 & 0 & 0 & 0 & 0 & 0 \\
0 & 0 & A_{2} & B_{2} & 0 & C_{2,1} & 0 & 0 \\
C_{3,2}^{T} & 0 & B_{2}^{T} & 0 & 0 & 0 & 0 & 0 \\
0 & 0 & 0 & 0 & A_{1} & B_{1} & 0 & C_{1,0} \\
0 & 0 & C_{2,1}^{T} & 0 & B_{1}^{T} & 0 & 0 & 0 \\
0 & 0 & 0 & 0 & 0 & 0 & I & 0 \\
0 & 0 & 0 & 0 & C_{1,0}^{T} & 0 & 0 & 0
\end{array}\right]\left[\begin{array}{c}
\boldsymbol{u}_{3} \\
p_{3} \\
\boldsymbol{u}_{2} \\
p_{2} \\
\boldsymbol{u}_{1} \\
p_{1} \\
\boldsymbol{u}_{0} \\
p_{0}
\end{array}\right]=\left[\begin{array}{c}
G_{3} \\
F_{3} \\
G_{2} \\
F_{2} \\
G_{1} \\
F_{1} \\
0 \\
0
\end{array}\right],
$$

where $\boldsymbol{u}_{d}$ and $p_{d}$ represent the vectors associated with the degrees of freedom of velocity and pressure in each dimension $d$. We note that the $\boldsymbol{u}_{0}$ is considered only for clearness and to preserve the structure of the matrix. In practice, it is possible to remove $\boldsymbol{u}_{0}$ from the system.

\subsection{Mixed-dimensional FV discretization}

We consider now the discretization of equation (4). For simplicity, we consider a fixed time step $\Delta t$ such that $T / \Delta t$ is an integer number. An implicit Euler is applied in time obtaining the semi-discrete version of (4)

$$
\begin{aligned}
& \frac{\left(\check{\phi} \check{\epsilon}\left(\check{c}^{n+1}-\check{c}^{n}\right), 1\right)_{E}}{\Delta t}+\left(\left.\check{\boldsymbol{u}} \cdot \check{\boldsymbol{n}}\right|_{\partial E},\left.\check{c}^{n+1}\right|_{\partial E}\right)_{\partial E} \\
& \quad+\sum_{ \pm}\left(\left.\check{\boldsymbol{u}} \cdot \check{\boldsymbol{n}}\right|_{I, \pm},\left.\check{c}^{n+1}\right|_{I, \pm}\right)_{I, \pm}-\left(\left.\hat{\boldsymbol{u}} \cdot \hat{\boldsymbol{n}}\right|_{E, \pm},\left.\hat{c}^{n+1}\right|_{E, \pm}\right)_{E} \\
& \quad=\left(\check{r}^{n+1}, 1\right)_{E}
\end{aligned}
$$

where the superscript indicates the time step index, and $c^{0}=\overline{\bar{c}}$. The approximation of the boundary integrals in the previous equation rely on an upwind scheme. We introduce a Kronecker-type delta as

$$
\delta_{u \cdot n}=\left\{\begin{array}{ll}
1 & \text { if } \boldsymbol{u} \cdot \boldsymbol{n} \geq 0 \\
0 & \text { else }
\end{array} .\right.
$$

Given a cell face $e \in \mathcal{E}(E)$ we have

$$
\begin{aligned}
& \left(\left.\check{\boldsymbol{u}} \cdot \check{\boldsymbol{n}}\right|_{e},\left.\check{c}^{n+1}\right|_{e}\right)_{e} \\
& \quad=\left.\check{\boldsymbol{u}} \cdot \check{\boldsymbol{n}}\right|_{e}\left[\delta_{\left.\check{u} \cdot \check{\boldsymbol{n}}\right|_{e}} \check{c}^{n+1}(E)+\left(1-\delta_{\left.\check{u} \cdot \check{\boldsymbol{n}}\right|_{e}}\right) \check{c}^{n+1}(L)\right],
\end{aligned}
$$

where the cells $K$ and $L$ share the face $e$. Given a cell $E$ such that one of its faces, $e$, intersects one side of $I$, we get

$$
\begin{aligned}
& \left(\left.\hat{\boldsymbol{u}} \cdot \hat{\boldsymbol{n}}\right|_{e},\left.\hat{c}^{n+1}\right|_{e}\right)_{e} \\
& \quad=\left.\hat{\boldsymbol{u}} \cdot \hat{\boldsymbol{n}}\right|_{e}\left[\delta_{\left.\hat{u} \cdot \hat{\boldsymbol{n}}\right|_{e}} \hat{c}^{n+1}(E)+\left(1-\delta_{\left.\hat{u} \cdot \hat{\boldsymbol{n}}\right|_{e}}\right) \check{c}^{n+1}(\check{e})\right],
\end{aligned}
$$

where $\check{e}$ indicates a cell in the co-dimensional grid which is in communication with the face $e$. Finally, on side giving an element $E$ of the last term of the semi-discrete problem can be approximated by

$$
\begin{aligned}
\left(\left.\hat{\boldsymbol{u}} \cdot \hat{\boldsymbol{n}}\right|_{E},\left.\hat{c}^{n+1}\right|_{E}\right)_{E} & \\
\quad=\left.\hat{\boldsymbol{u}} \cdot \hat{\boldsymbol{n}}\right|_{E}\left[\delta_{\left.\hat{u} \cdot \hat{n}\right|_{e}} \hat{c}^{n+1}(\hat{E})+\left(1-\delta_{\left.\hat{u} \cdot \hat{\boldsymbol{n}}\right|_{e}}\right) \check{c} n+1\right. & (E)],
\end{aligned}
$$

where $\hat{E}$ represents the cell in the higher dimensional grid which has a face $e$ in communication with the cell $E$. The previous condition applies to both the equi- and co-dimensional coupling, see Figure 4 for an example. Note that the chosen discretization is compatible with the coupling condition $(2 \mathrm{~b})$.

For the matrix formulation of the transport problem, we introduce the following matrices

$$
\begin{gathered}
{\left[M_{d}\right]_{i i}=\frac{\left(\phi^{d} \epsilon^{d}, 1\right)_{i}}{\Delta t}, \quad\left[U_{d}\right]_{i i}=\sum_{j \in \mathcal{N}(i), e \in \bar{i} \cap \bar{j}}\left(\left.\boldsymbol{u}^{d} \cdot \boldsymbol{n}^{d}\right|_{e}, \delta_{\left.\boldsymbol{u}^{d} \cdot \boldsymbol{n}^{d}\right|_{e}}\right)_{e},} \\
{\left[U_{d}\right]_{i j}=\sum_{j \in \mathcal{N}(i), e \in \bar{i} \cap \bar{j}}\left(\left.\boldsymbol{u}^{d} \cdot \boldsymbol{n}^{d}\right|_{e}, 1-\delta_{\left.u^{d} \cdot \boldsymbol{n}^{d}\right|_{e}}\right)_{e},}
\end{gathered}
$$

where $\mathcal{N}(E)$ is the set of all neighbor cells of $E, i$ and $j$ indicate generic cells and $e$ is a face shared by $i$ and $j$. For the coupling between dimensions we have

$$
\left[U_{d, d-1}\right]_{i i}=\sum_{j \in \mathcal{N}^{d-1}(i)}\left(\left.\boldsymbol{u}^{d} \cdot \boldsymbol{n}^{d}\right|_{j}, \delta_{\left.u^{d} \cdot n^{d}\right|_{j}}\right)_{j},
$$



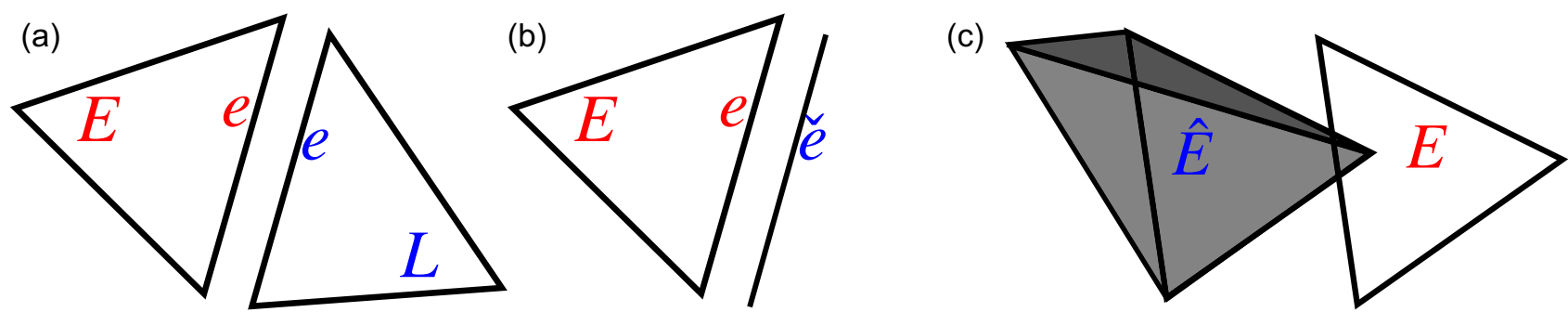

Fig. 4. Representation of the coupling between dimension for the upwind discretization scheme. (a) Between two cells in the same dimension $d$. (b) Between a $d$-dimensional cell and a $d$-1-dimensional cell. (c) Between a $d+1$-dimensional cell and a $d$-dimensional cell.

$$
\left[U_{d, d-1}\right]_{i j}=\sum_{j \in \mathcal{N}^{d-1}(i)}\left(\left.\boldsymbol{u}^{d} \cdot \boldsymbol{n}^{d}\right|_{j}, 1-\delta_{\left.u^{d} \cdot n^{d}\right|_{j}}\right)_{j}
$$

where $\mathcal{N}^{d-1}(E)$ is the set of all neighbor cells of $E$ in the lower-dimensional grid. We obtain also

$$
\begin{gathered}
{\left[U_{d, d+1}\right]_{i i}=-\sum_{j \in \mathcal{N}^{d+1}(i)}\left(\left.\boldsymbol{u}^{d+1} \cdot \boldsymbol{n}^{d+1}\right|_{i}, \delta_{\left.u^{d+1} \cdot \boldsymbol{n}^{d+1}\right|_{i}}\right)_{i},} \\
{\left[U_{d, d+1}\right]_{i j}=-\sum_{j \in \mathcal{N}^{d+1}(i)}\left(\left.\boldsymbol{u}^{d+1} \cdot \boldsymbol{n}^{d+1}\right|_{i}, 1-\delta_{\left.u^{d+1} \cdot n^{d+1}\right|_{i}}\right)_{i},}
\end{gathered}
$$

where $\mathcal{N}^{d+1}(E)$ is the set of all neighbor cells of $E$ in the higher-dimensional grid. We finally obtain the following linear system to be inverted

$$
\begin{aligned}
& {\left[\begin{array}{cccc}
U_{3}+M_{3} & U_{3,2} & 0 & 0 \\
U_{2,3} & U_{2}+M_{2} & U_{2,1} & 0 \\
0 & U_{1,2} & U_{1}+M_{1} & U_{1,0} \\
0 & 0 & U_{0,1} & 0
\end{array}\right]} \\
& \times\left[\begin{array}{c}
c_{3}^{n+1} \\
c_{2}^{n+1} \\
c_{1}^{n+1} \\
c_{0}^{n+1}
\end{array}\right]=\left[\begin{array}{c}
\zeta_{3}^{n, n+1} \\
\zeta_{2}^{n, n+1} \\
\zeta_{1}^{n, n+1} \\
0
\end{array}\right],
\end{aligned}
$$

where $c_{d}$ represents the vector associated with the degrees of freedoms of the concentration in each dimension $d$. In the previous linear system $\zeta_{d}$ represents the right-hand side, formed as a combination of the source term $r_{d}^{n+1}$ and the concentration at the previous time step. We get

$$
\zeta_{d}^{n, n+1}=M_{d} c_{d}^{n}+r_{d}^{n+1} .
$$

\subsection{Coarsening strategy}

The construction of conforming grids in the presence of several fractures can be a challenging task, especially in 3d. In this work, our grids are constructed by the Gmsh mesh generator [49]. In the presence of almost intersecting fractures or small fracture branches, the grid may contain a high number of simplex cells, resulting in a high computational cost. To overcome this difficulty, we exploit the ability of VEM to handle cells of arbitrary geometry. To be specific, the theory developed in [30-33] requires star-shaped cells, however the study carry out in [50] shows that the VEM is able to handle also cells with cuts.

Motivated by these observations we introduce a coarsening scheme that merges small simplex cells into larger polygons or polyhedra. Starting from a given simplex grid, the algorithm computes the measure (area or volume) of the cells. Given the cell $c$ with the smallest measure, it will be merged to one or more neighboring cells, based on their respective measure, creating a new coarse cell. The clustering stops when a cell measure threshold is reached. The cells used in discretization are thus general polytopes, formed as unions of simplexes.

The algorithm does not guarantee any regularity of the final grid, as illustrated in Figure 5.

We emphasize that as elements are aggregated, a new element with many faces is generated. The local matrices will be denser and larger comparing to the original grid and increase the stencil of the global matrix. Nevertheless, the coarsening algorithm will decrease the computational cost. Other strategies are possible but not considered in this work.

Finally, we note that the assumption of a cell-wise constant permeability applies also in this case. Thus, merging of cells with different permeabilities requires the computation of an average permeability. Correspondingly, for highly heterogeneous media, it may be beneficial to consider also cell permeability as a parameter in the coarsening algorithm.

\section{Applicative examples}

We present three examples and test cases to assess the presented models and numerical schemes. The first example, presented in Section 5.1, considers an extensive validation of the mixed-dimensional Darcy problem solved by VEM through a benchmark study presented in [51]. The second test case, in Section 5.2, considers the transport problem and analyzes the impact of the coarsening strategy on the results. Finally, in Section 5.3 a realistic 3d example is introduced and studied. In all the forthcoming examples we assume unitary porosity and zero source terms for the concentration and Darcy equations. The other parameters will be specified. 
(a)
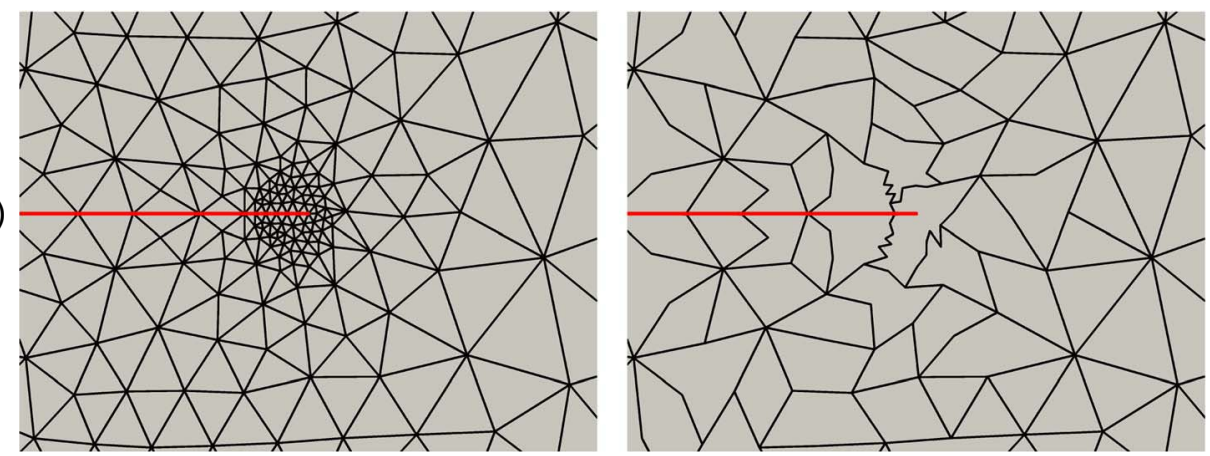

(b)

Fig. 5. Example of the coarsening strategy adopted. (a) The computational grid is artificially forced to be finer at the tip of a fracture. (b) The resulting grid after the coarsening. Clearly the cell measures are comparable.

In all of the forthcoming examples a "Blue to Red Rainbow" color map is used.

The examples are part of the PorePy package, which is a simulation tool for fractured and deformable porous media written in Python. See [52] and github.com/pmgbergen/porepy for more details.

\subsection{Benchmark comparison}

To validate the presented model, we consider the benchmark study presented in [51], specifically cases 1 and 4 . The reference solutions $p^{\text {ref }}$ were computed with a mimetic finite difference method [53] on a very fine grid that is able to represent the thickness of the fractures by considering the classical Darcy model. The error is thus evaluated by

$$
\begin{aligned}
& e r r^{2}=\sqrt{\frac{1}{\left|\Omega^{2}\right| \Delta^{\text {ref }}} \sum_{E=E^{\text {ref }} \cap E^{2}}|E|\left(\left.p^{2}\right|_{E^{2}}-\left.p^{\text {ref }}\right|_{E^{\text {ref }}}\right)^{2}}, \\
& e r r^{1}=\sqrt{\frac{1}{\left|\Omega^{1}\right| \Delta^{\text {ref }}} \sum_{E=E^{\text {ref }} \cap E^{1}}|E|\left(\left.p^{1}\right|_{E^{1}}-\left.p^{\text {ref }}\right|_{E^{\text {ref }}}\right)^{2}},
\end{aligned}
$$

for the matrix $e r r^{2}$ and for the fractures $e r r^{1}$, where $\Delta^{\text {ref }}=\left(\max p^{\text {ref }}-\min p^{\text {ref }}\right)^{2}$ and $E^{2}$ is a cell in the mixed-dimensional problem related to the rock matrix, respectively $E^{1}$ for the fracture. The errors are computed on the reference grid, $p^{1}$ is assumed constant on the normal direction of the fracture in the equi-dimensional setting.

For more detail of problem setting refer to the aforementioned work.

\subsubsection{Benchmark 1: regular fracture network}

The problem is inspired by Geiger et al. [54] with different boundary conditions and material properties. The domain is the unit square containing six fractures, sketched in Figure 6. The matrix permeability is taken as unitary, and the fracture aperture is set equal to $10^{-4}$. No flux boundary conditions are imposed on the top and bottom of the domain, while unitary pressure is imposed on the right boundary and -1 as flux on the left boundary.

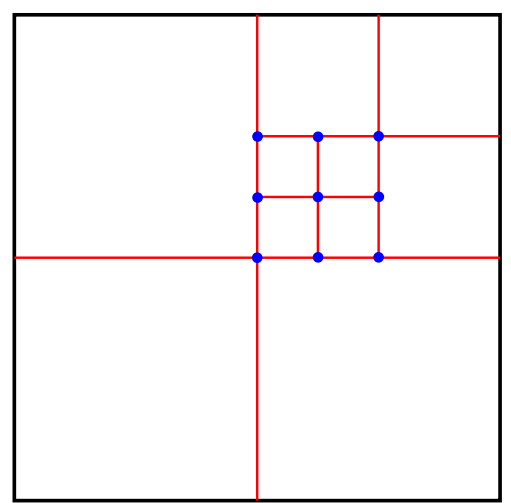

Fig. 6. Domain with fractures (in red) and fracture intersections (in blue) for problem in Section 5.1.1.

The source term is set to zero. We consider two possibilities for fracture permeability: it is either highly conductive with permeability $10^{4}$ and has conductivity with permeability $10^{-4}$. In the former case the solution obtained with the method presented previously along with the computational grid are presented in Figure 7(a), the latter in (b). We observe a good agreement between the computed and reference solutions, the latter is described in [51]. We point out that some of the elements present in Figure 7 are not convex.

To obtain a more detailed comparison we consider two plots over line for the permeable case and one for the blocking case, shown in Figure 8. From now on, the method presented in this paper is labeled as VEM. Also in this case, the agreement between the computed and reference solutions is comparable to the other methods which are able to represent blocking fractures. The small oscillations in the line plots are related to grid effects.

Finally, in both cases we consider the error decay for both the rock matrix and the system of fractures. We consider a family of three grids where the coarsening is applied in all the cases. We obtain again non-convex elements in all the grids. Figure 9 plots for conductive and blocking fractures the error decay. The errors are comparable with those of others methods able to represent permeable and blocking fractures. In the latter case we note a stagnation of the 
(a)

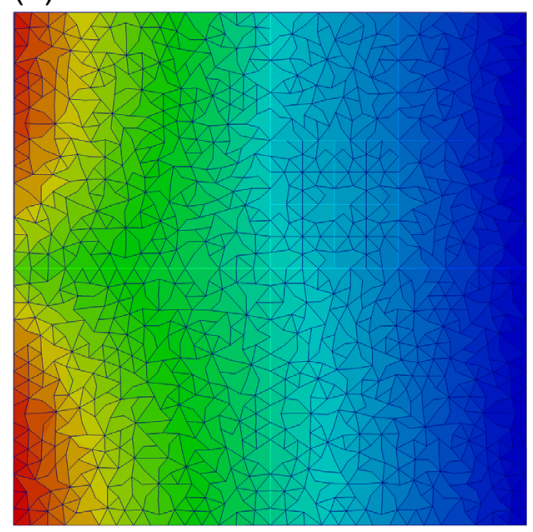

(b)

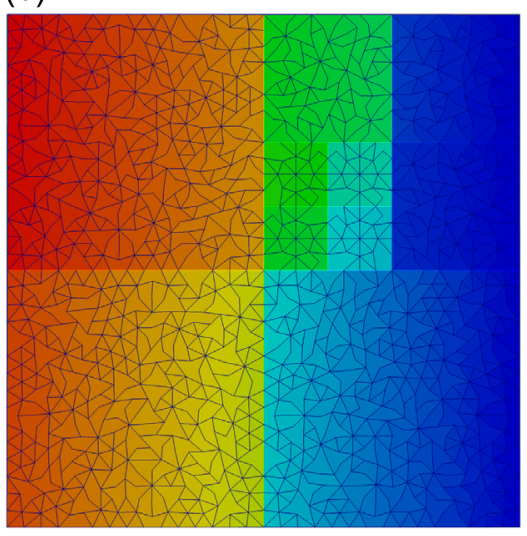

(c)

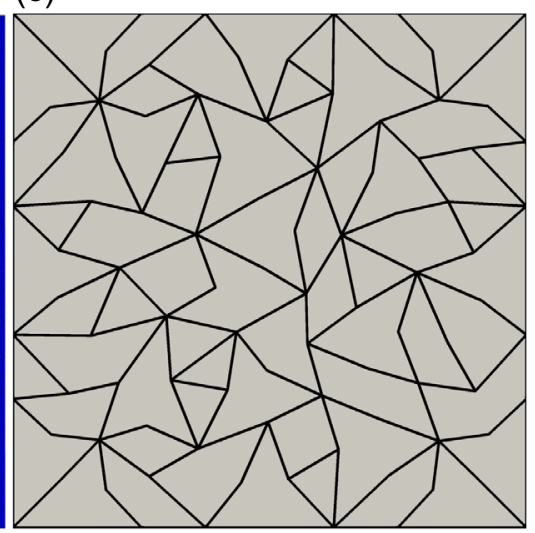

Fig. 7. Benchmark 1. (a) Pressure solution (range $(1,1.6)$ ) with conductive fractures and computational grid. (b) Pressure solution (range $(1,3.6))$ with blocking fractures and computational grid. (c) Zoom of the grid used.
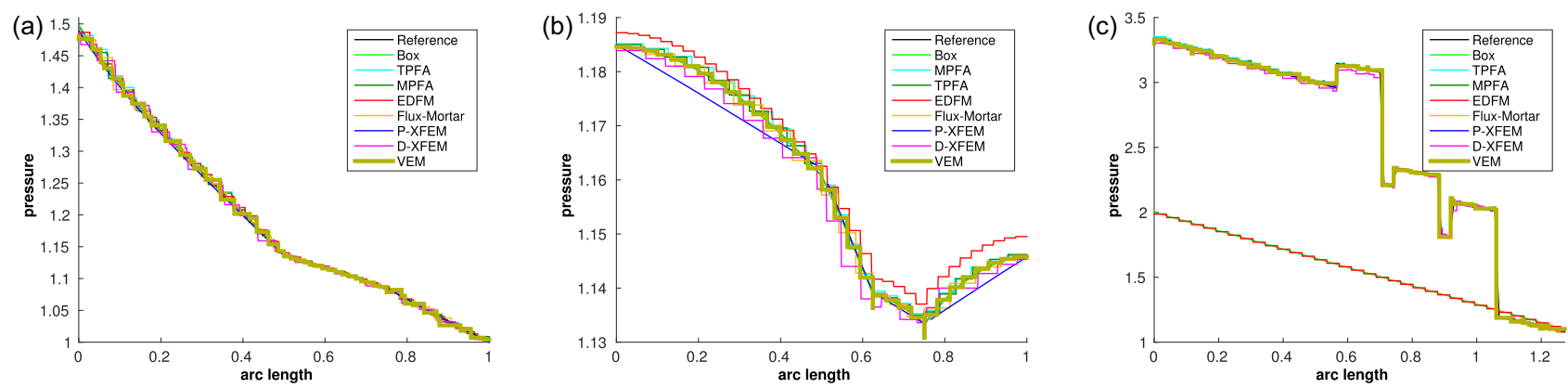

Fig. 8. Benchmark 1 with conductive fractures. (a) Pressure along horizontal line at $y=0.7$ with permeable fracture. (b) Pressure along vertical fracture at $x=0.5$ with permeable fractures. (c) Pressure along the line $(0.0,0.1)-(0.9,1.0)$ with blocking fractures.
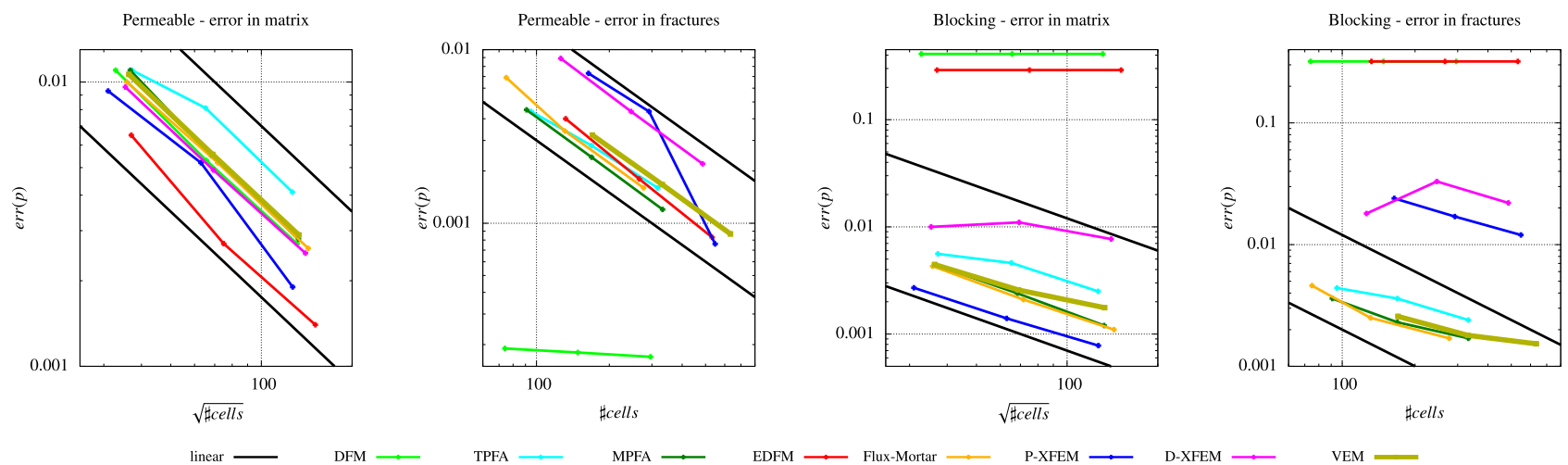

Fig. 9. Benchmark 1, error evolution for the matrix and the fractures with conductive and blocking fractures.

fracture error which bounds the order of converge. This phenomenon is common also for other methods presented in the benchmark study [51].

\subsubsection{Benchmark 4: a realistic case}

We consider now a complex system of 64 fractures from a real outcrop. The domain is $\Omega=(0,700 \mathrm{~m}) \times(0,600 \mathrm{~m})$, sketched in Figure 10. We consider constant rock permeability equal to $10^{-14} \mathrm{~m}^{2}$, uniform fracture permeability $10^{-8} \mathrm{~m}^{2}$, and fracture aperture $10^{-2} \mathrm{~m}^{2}$. We impose a pressure gradient at the boundary from the left (1 $013250 \mathrm{~Pa})$ to the right $(0 \mathrm{~Pa})$, and no flow condition on the top and bottom of the domain. The source term is set to zero. Also in this case, a triangular grid is coarsened to reduce the grid complexity. The reference grid is composed by $124722 \mathrm{~d}$ 


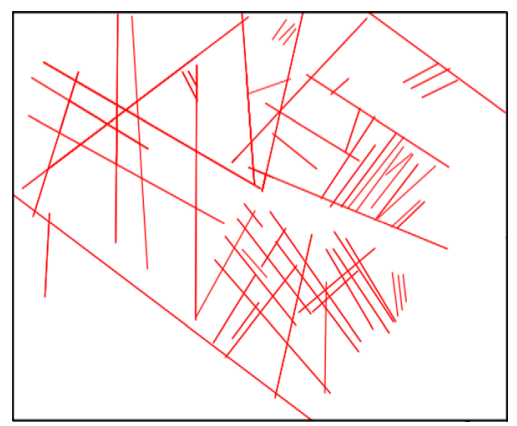

Fig. 10. Domain with fractures (in red) for problem in Section 5.1.2.

cells, 1317 1d cells, and 85 0d cells. The coarse algorithm decreases the number of $2 \mathrm{~d}$ cells down to 4703 . The total number of degrees of freedom is 19075 for the coarse grid.

The computed solution is represented in Figure 12(a), which matches the solution of the others method considered in [51]. Because of the complexity of the network, conforming and non-matching methods may pose a constraint to the grid generation in particular for close fractures. However, the method presented allows general grid cells, and thus alleviate the computational cost for the simulation. For an illustration of the computational grid, we refer to Figure 11, which zooms in on a region with almost intersecting fractures.

As shown in Figure 11, some of the grid cells are non starshaped or even contains cuts. For a more detailed discussion refer to [50]. Finally, to validate in more detail the computed solution we present two plots over line and compare them with the solutions obtained in the benchmark study, see Figure 12. The curves for the current method are in good agreement with the others. Again, the small oscillations that can be observed are related to grids effects.

\subsection{Passive scalar transport}

In this part we consider both mixed-dimensional models (1) and $(2)$ to simulate a passive scalar transport. We consider the geometry presented in Section 5.1.2 and compare the solution obtained with both grids in Figure 11 for permeable and blocking fractures. For the reference grid, the total number of degrees of freedom is 35578 . The aim of this test is to validate the quality of the Darcy velocity on the passive scalar in presence of the grid coarsening. In the following fracture aperture is constant and equal to $10^{-2} \mathrm{~m}$, a pressure gradient from the right to left boundary of the domain of $3 \times 10^{7} \mathrm{~Pa}$, a final simulation time of 40 years.

\subsubsection{Permeable fractures}

We consider highly permeable fractures with tangential permeability of $5 \times 10^{-6} \mathrm{~m}^{2}$ and normal permeability of $2.5 \times 10^{-9} \mathrm{~m}^{2}$. The matrix permeability is set to $2.5 \times$ $10^{-11} \mathrm{~m}^{2}$. Figure 13 compares the solutions obtained on the reference triangular grid and on the coarse grid. Moreover Figure 14(a) presents a comparison of the passive scalar production. We notice the good agreement in both the pressure and concentration fields as well as in the production curve. We can conclude that in this case the grid coarsening is not affecting the quality of the computed solutions.

Finally, in Figure 14(b) the temporal error decay is reported. The spatial discretization is fixed and we consider a sequence of simulation with $(10,20,40,80,160,320,640$, (a)

(b)
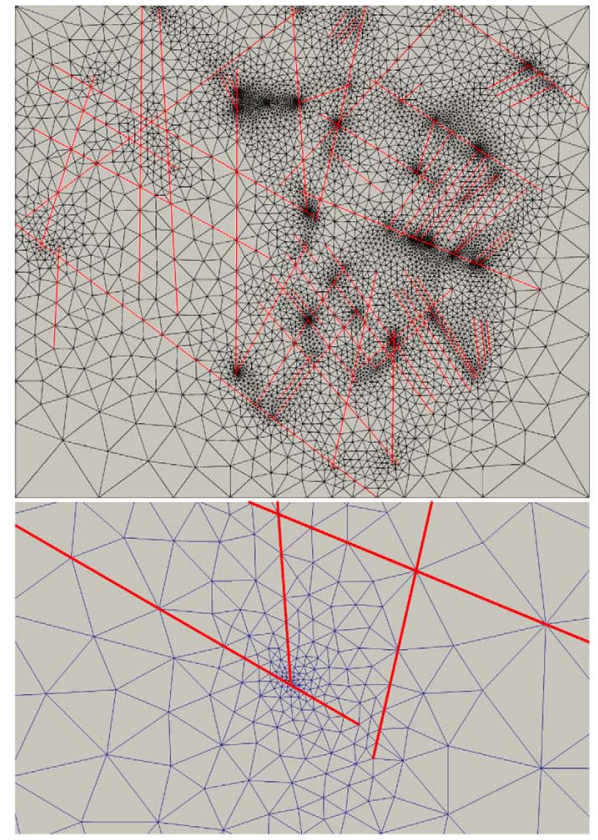

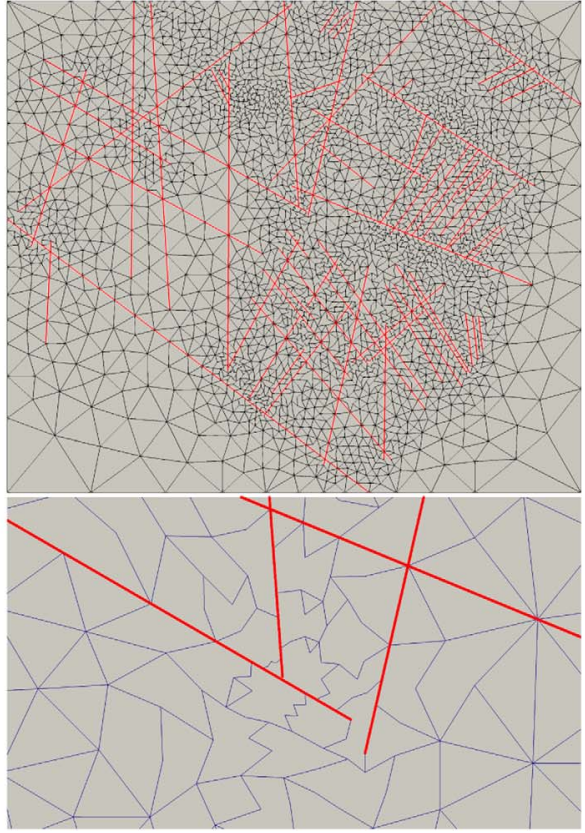

Fig. 11. Benchmark 4. (a) Original grid composed by 12302 2d-cells and 35153 VEM dof and coarsened grid composed by 4599 2d-cells and 18803 VEM dof. (b) A zoom on almost intersecting fractures for the original and coarsened grids, respectively. The zoom is referred to the small rectangle at position, approximately $(360,350)$. 

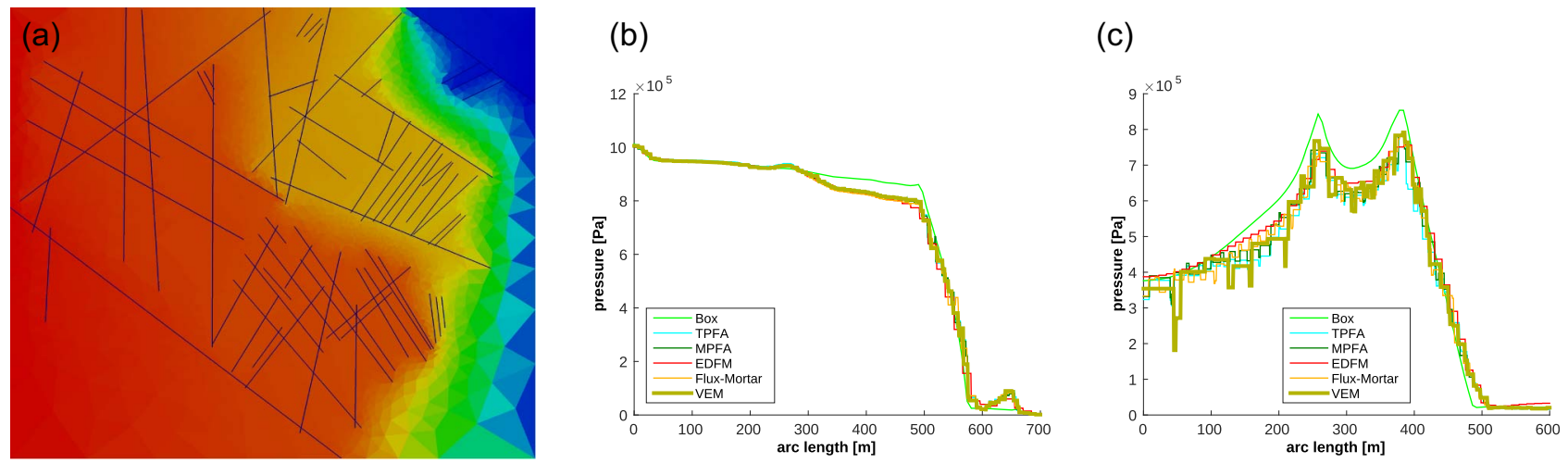

Fig. 12. Benchmark 4. (a) Pressure solution computed with VEM. The pressure ranges in [0, 1 013 250] Pa. (b) Pressure along horizontal line at $y=500 \mathrm{~m}$. (c) Pressure along vertical line at $x=625 \mathrm{~m}$.

(a)
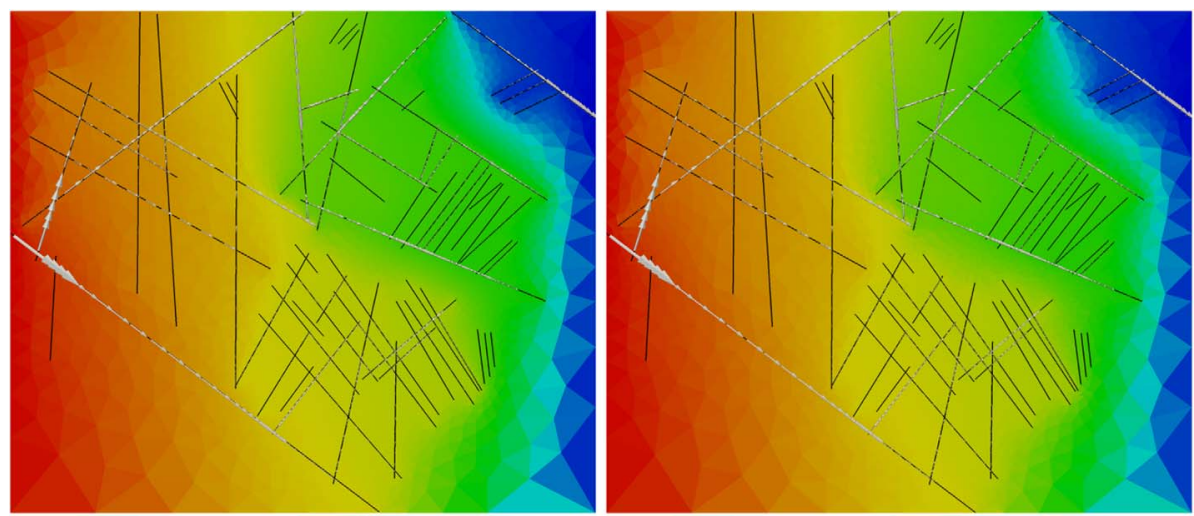

(b)
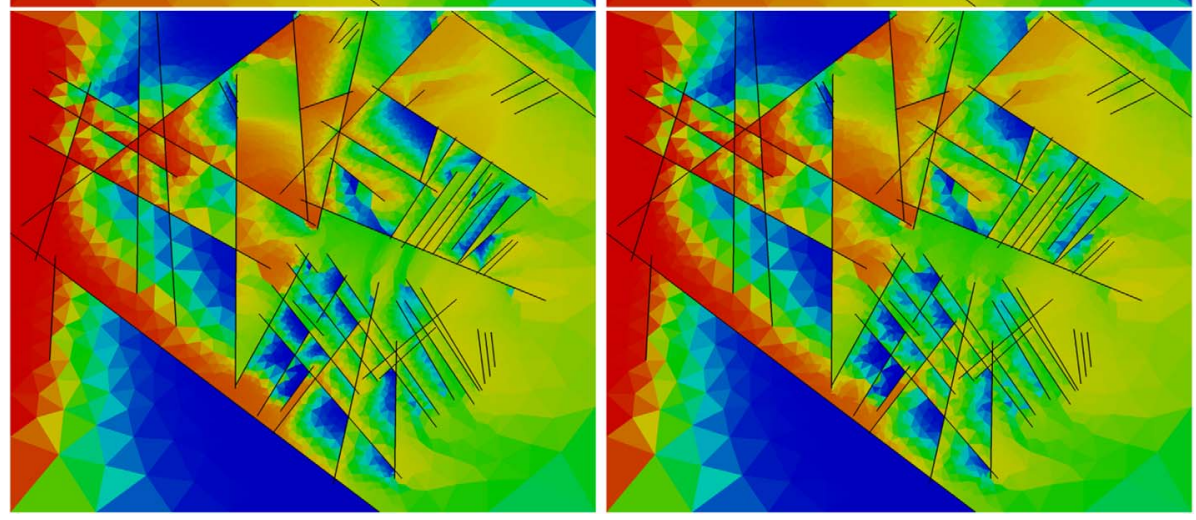

Fig. 13. Permeable fractures. (a) Reference and coarse solutions for pressure and velocity, as arrows. (b) Reference and coarse solutions for concentration of the passive scalar.

$1280,2560,5120)$ time steps each. The error is computed as the $L^{2}$ difference from a reference solution obtained with $10^{5}$ time steps after 10 years. A unitary error decay is achieved, coherent with the numerical scheme considered.

\subsubsection{Blocking fractures}

Next, we consider blocking fractures with tangential and normal permeability of $7.5 \times 10^{-16} \mathrm{~m}^{2}$. Matrix permeability is set to $7.5 \times 10^{-11} \mathrm{~m}^{2}$. Figure 15 compares the solutions obtained on the reference triangular grid and on the coarse grid. In this case we notice a pickpeak of velocity in the reference case due to small elements close to almost intersecting fractures. This may affect an explicit in time solver. Figure 16(a) presents a comparison of the passive scalar production. We notice the good agreement in both the pressure and concentration fields as well as in the production. We can conclude that also in this case the grid coarsening is not affecting the quality of the computed solutions.

Again, the temporal error decay is reported, see Figure 16(b). The spatial discretization is fixed and we assign $(10,20,40,80,160,320,640,1280,2560,5120)$ time 

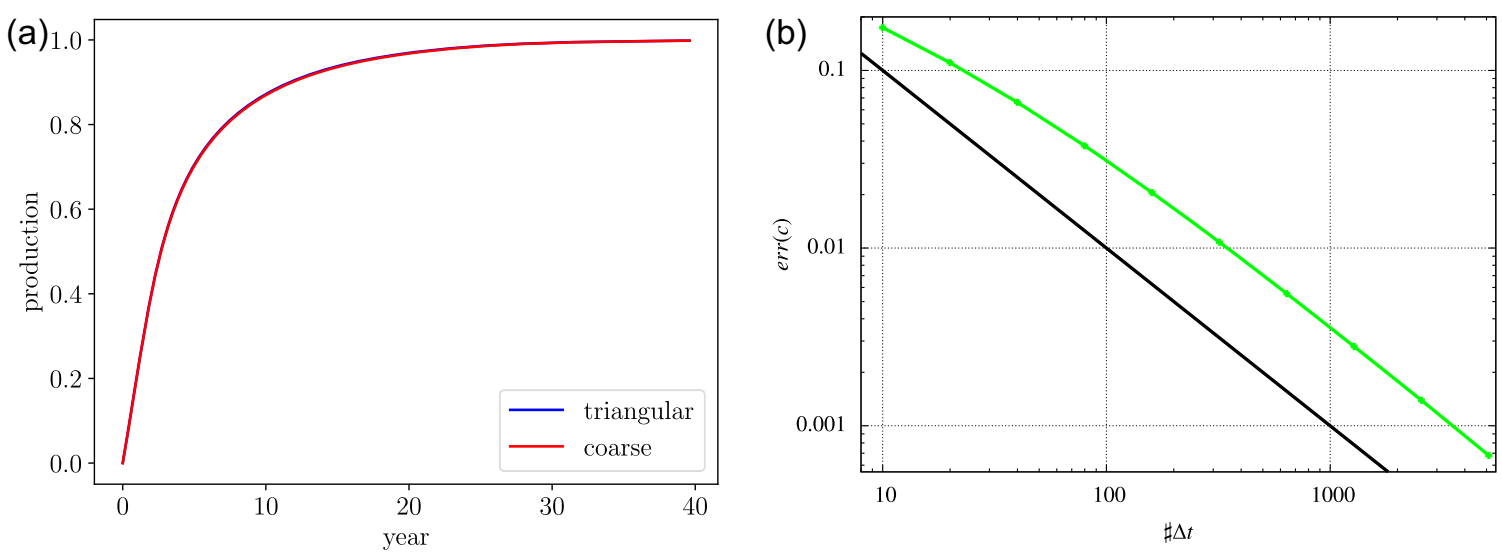

Fig. 14. Permeable fractures. (a) Comparison of passive scalar production at the outflow between the reference triangular grid and the coarse grid. (b) Temporal error decay with reference $\mathcal{O}(\Delta t)$ in black.

(a)
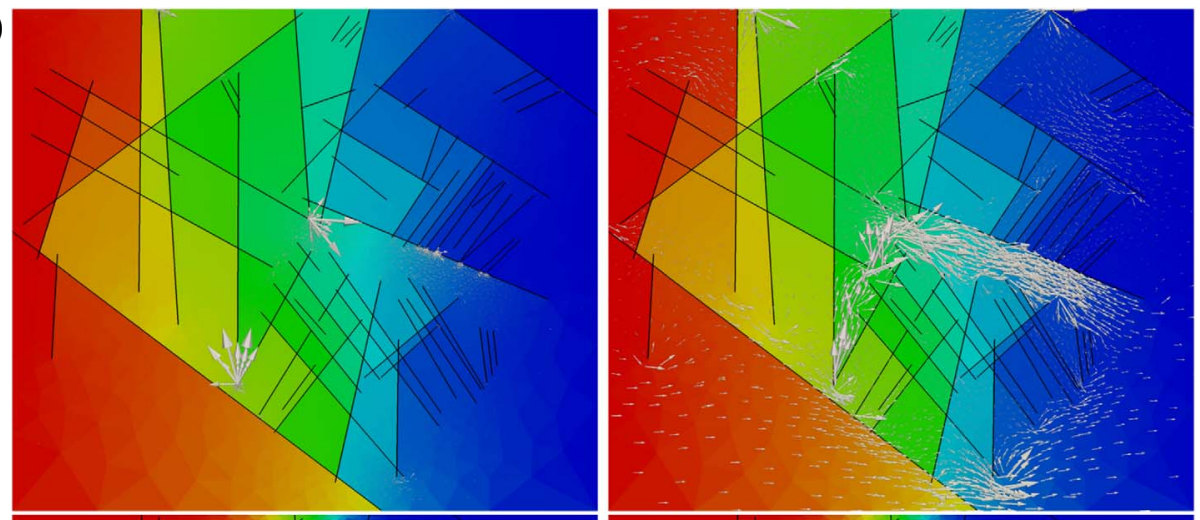

(b)
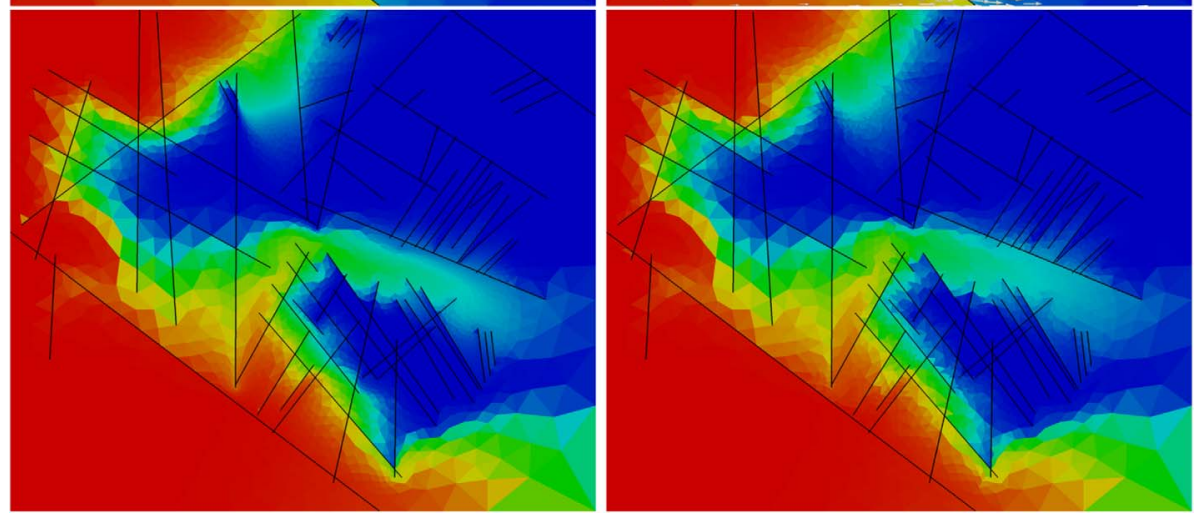

Fig. 15. Blocking fractures. (a) Reference and coarse solutions for pressure and velocity, as arrows. (b) Reference and coarse solutions for concentration of the passive scalar.

steps, respectively. A unitary error decay is achieved, consistent with the numerical scheme considered.

\subsection{Passive scalar transport on a realistic 3d-network}

In this example we consider a realistic geometry for a geothermal system. We study a partial reconstruction of fractures from test site at Soultz-sous-Forêts in France, for more details see [55]. The network is composed by 20 fractures represented as polygons with 10 edges each. The fracture intersections result in $331 \mathrm{~d}$ objects and $40 \mathrm{~d}$ objects. In this case the full model is needed to accurately simulate the fluid flow and transport on the domain. The fracture geometry is represented in Figure 17. We assume rock matrix permeability as $7.5 \times 10^{-10} \mathrm{~m}^{2}$ and fracture permeability, in both the normal and tangential direction, equal to $5 \times 10^{-5} \mathrm{~m}^{2}$. The fracture aperture is $\epsilon^{2}=10^{-2} \mathrm{~m}$ and, for the lower dimensional objects, we consider their "aperture" as the square 

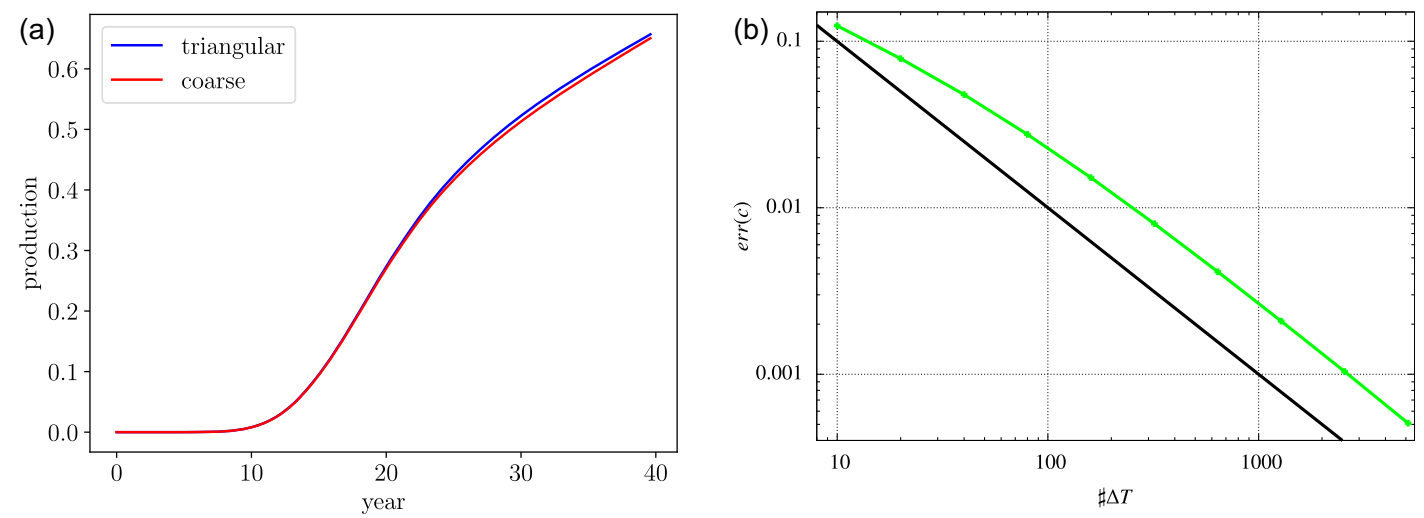

Fig. 16. Blocking fractures. (a) Comparison of passive scalar production at the outflow between the reference triangular grid and the coarse grid. (b) Temporal error decay with reference $\mathcal{O}(\Delta t)$ in black.
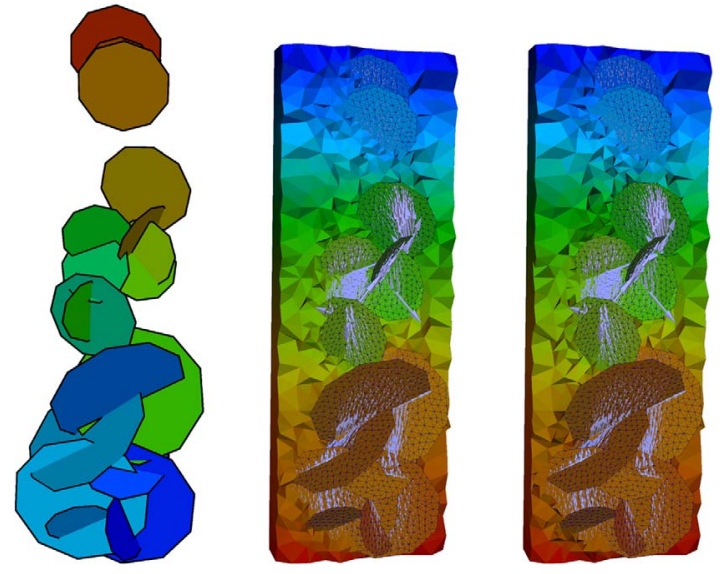

Fig. 17. On the left representation of the 20 fractures colored by their identification number. Pressure and velocity for both the reference and coarse grids. The pressure is scaled between 0 and $4.8 \times 10^{7} \mathrm{~Pa}$. and the cube of the fracture aperture, respectively for the $1 \mathrm{~d}$ and 0d objects. We impose a pressure from the top to bottom of the domain and no flux boundary conditions on the other sides. The reference grid is composed by 44331 tetrahedra, 6197 triangles for the 2 d grids, 151 segments for the 1d grids, and 4 point-cells for the 0d grids. After the coarsening algorithm the resulting grid is composed by 16108 polyhedra, and for the lower dimensional objects the grids are untouched. See Figure 19(b) for an example of coarse cells. The transport simulation runs for 40 years and, for the implicit Euler scheme, we consider 100 time steps.

The objective is to study the robustness of the virtual elements associated with the coarsening strategy and detect if the coarse model gives accurately enough results. The pressure solution and the concentration of the scalar passive are depicted in Figures 17 and 18, respectively, for both the reference and coarse case. Both the pressure and concentration profiles for the two grids are in good agreement. To analyze the macroscopic behavior of the resulting solution
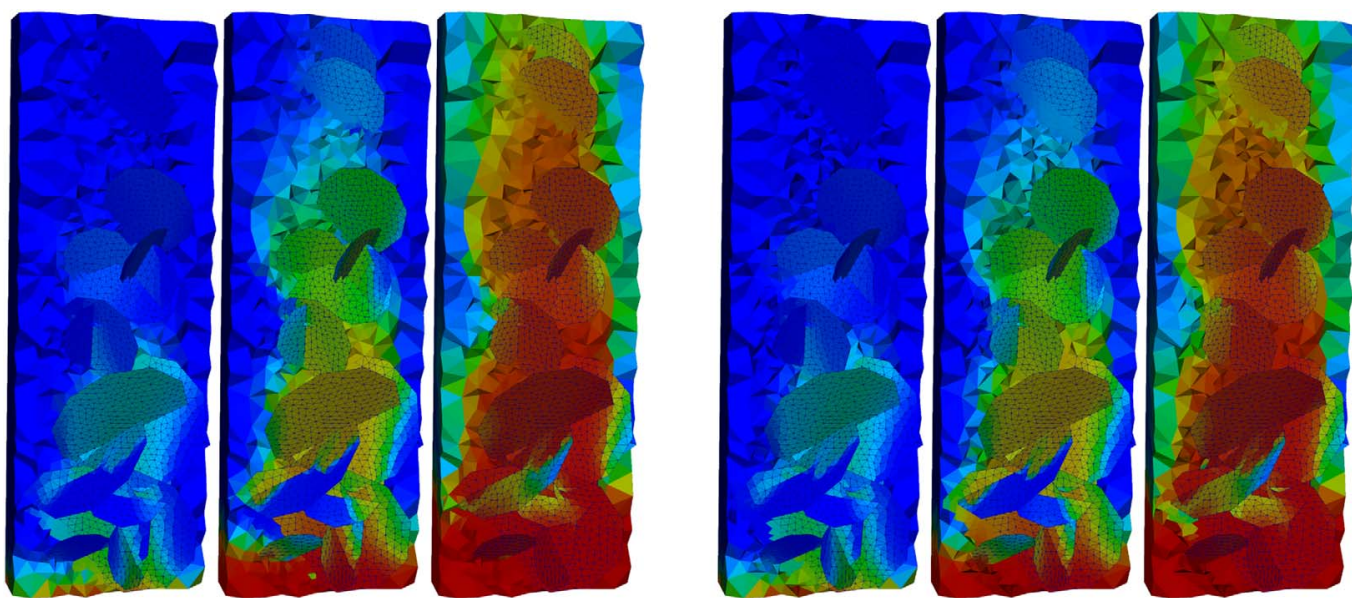

Fig. 18. (a) Three time steps on the reference grid for the concentration. (b) At the same steps, the concentration computed on the coarse grid. 
(a)

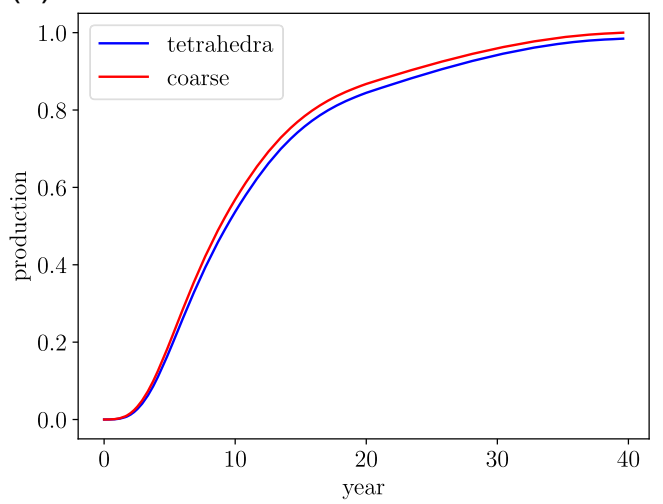

(b)

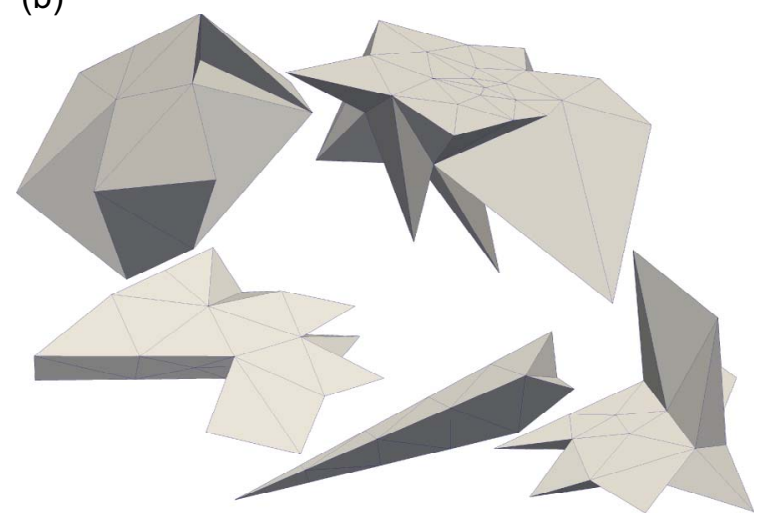

Fig. 19. (a) The production comparison between the reference and the coarse solution. (b) An example of five coarse cells.

a production curve comparison is reported in Figure 19, showing a small discrepancy from the reference and the coarse production.

We can conclude that also in this case the adopted strategy is effective and can be applied to complex system of fractures, ensuring a good compromise between high accuracy and computational effort.

\section{Conclusion}

In the paper we presented two classes of mixed-dimensional problems able to describe a single-phase flow and a transport of a scalar passive in fractured porous media. The latter is transported by the Darcy velocity computed by the former model. The considered mixed-dimensional Darcy problem is able to represent both channel and barrier behaviors of fractures, as well as their intersections. The models thus represent a comprehensive description of this phenomena, which are key ingredients for the description of several energy application problems. The numerical schemes considered for the discretization of the Darcy equation are able to handle, supported by theoretical findings, grid cells of arbitrary geometry becoming a strong advantage when dealing with high and complex fractured porous media. Numerical results have shown the capability to apply this strategy obtaining accurate outcomes with a reasonable computational cost. The transport model can be viewed as a first attempt to introduce a full model for the description of heat exchange in a porous media, which will be a part of future investigations.

Acknowledgments. We acknowledge financial support for the ANIGMA project from the Research Council of Norway (project no. 244129 (E20) through the ENERGIX program. The authors warmly thank the others components of the ANIGMA project team: Eivind Bastesen, Inga Berre, Simon John Buckley, Casey Nixon, David Peacock, Atle Rotevatn, Pål Næverlid Sævik, Luisa F. Zuluaga.

The authors wish to thank also: Runar Berge, Wietse Boon, Ivar Stefansson, Eren Uçar.

\section{References}

1 Berre I., Boon W., Flemisch B., Fumagalli A., Gläser D., Keilegavlen E., Scotti A., Stefansson I., Tatomir A. (2018) Call for participation: Verification benchmarks for singlephase flow in three-dimensional fractured porous media. Technical report, arXiv:1710.00556 [math.AP].

2 Mourzenko V.V., Thovert J.-F., Adler P.M. (Sep 2011) Permeability of isotropic and anisotropic fracture networks, from the percolation threshold to very large densities, Phys. Rev. E 84, 036307. https://doi.org/10.1103/PhysRevE. URL https://link.aps.org/doi/10.1103/PhysRevE.84.036307.

3 Sævik P.N., Berre I., Jakobsen M., Lien M. (Oct 2013) A 3d computational study of effective medium methods applied to fractured media, Transp. Porous Media, 100, 115-142. ISSN 1573-1634. https://doi.org/10.1007/s11242-013-0208-0.

4 Ssvik P.N., Jakobsen M., Lien M., Berre I. (2014) Anisotropic effective conductivity in fractured rocks by explicit effective medium methods, Geophys. Prospect. 62, 6, 12971314. ISSN 1365-2478. https://doi.org/10.1111/1365-2478. 12173.

5 Fumagalli A., Pasquale L., Zonca S., Micheletti S. (2016) An upscaling procedure for fractured reservoirs with embedded grids, Water Resour. Res. 52, 8, 6506-6525. ISSN 1944-7973. https://doi.org/10.1002/2015WR017729.

6 Karimi-Fard M., Durlofsky L.J. (Oct 2016) A general gridding, discretization, and coarsening methodology for modeling flow in porous formations with discrete geological features, $A d v$. Water Res. 96, 354-372. ISSN 03091708. https://doi.org/ 10.1016/j.advwatres.2016.07.019. URL http://linkinghub. elsevier.com/retrieve/pii/S0309170816302950.

7 Karimi-Fard M., Gong B., Durlofsky L.J. (2006) Generation of coarse-scale continuum flow models from detailed fracture characterizations, Water Resour. Res. 42, 10. ISSN 1944-7973. https://doi.org/10.1029/2006WR005015.

8 Alboin C., Jaffré J., Roberts J.E., Wang X., Serres C., Chen Z., Ewing R. E., Shi Z.-C. (2000) Domain decomposition for some transmission problems in flow in porous media. Numerical treatment of multiphase flows in porous media (Beijing, 1999), Springer, pp. 22-34.

9 Amir L., Kern M., Martin V., Roberts J.E. (2005) Décomposition de domaine et préconditionnement pour un modèle $3 \mathrm{D}$ en milieu poreux fracturé, in: Proceeding of JANO 8, $8^{\text {th }}$ 
Conference on Numerical Analysis and Optimization, December 2005.

10 Boon W.M., Nordbotten J.M., Yotov I. (2018) Robust discretization of flow in fractured porous media, SIAM J. Numer. Anal. 56, 4, 2203-2233. https://doi.org/10.1137/ 17M1139102.

11 Faille I., Fumagalli A., Jaffré J., Roberts J.E. (2016) Model reduction and discretization using hybrid finite volumes of flow in porous media containing faults, Comput. Geosci. 20, 2, 317-339. ISSN 15731499. https://doi.org/10.1007/s10596016-9558-3.

12 Knabner P., Roberts J.E. (2014) Mathematical analysis of a discrete fracture model coupling Darcy flow in the matrix with Darcy-Forchheimer flow in the fracture, ESAIM: Math. Model. Numer. Anal. 48, 1451-1472. ISSN 1290-3841. https://doi.org/10.1051/m2an/2014003. URL http://www. esaim-m2an.org/article_S0764583X1400003X.

13 Martin V., Jaffré J., Roberts J.E. (2005) Modeling fractures and barriers as interfaces for flow in porous media, SIAM J. Sci. Comput. 26, 5, 1667-1691. ISSN 1064-8275. https://doi. org/10.1137/S1064827503429363. URL http://scitation.aip. org/getabs/servlet/GetabsServlet?prog=normal\&id= SJ0CE3000026000005001667000001\&idtype $=$ cvips\&gifs $=$ yes.

14 Schwenck N., Flemisch B., Helmig R., Wohlmuth B.I. (2015) Dimensionally reduced flow models in fractured porous media: crossings and boundaries, Comput. Geosci. 19, 6, 1219-1230. ISSN 1420-0597. https://doi.org/10.1007/ s10596-015-9536-1.

15 Tunc X., Faille I., Gallouet T., Cacas M.C., Havé P. (2012) A model for conductive faults with non-matching grids, Comput. Geosci. 16, 277-296. ISSN 1420-0597. https://doi. org $/ 10.1007 /$ s10596-011-9267-x.

16 Angot P. (2003) A model of fracture for elliptic problems with flux and solution jumps, C. R. Math. 337, 6, 425-430. ISSN 1631-073X. https://doi.org/10.1016/S1631-073X(03)00300-5. URL http://www.sciencedirect.com/science/article/pii/ S1631073X03003005.

17 Angot P., Boyer F., Hubert F. (2009) Asymptotic and numerical modelling of flows in fractured porous media, M2AN Math Model. Numer. Anal. 43, 2, 239-275. ISSN 0764-583X. https://doi.org/10.1051/m2an/2008052.

18 Chave F.A., Di Pietro D., Formaggia L. (2017) A Hybrid High-Order method for Darcy flows in fractured porous media, Technical report, HAL archives, 2017. URL https:// hal.archives-ouvertes.fr/hal-01482925.

19 Karimi-Fard M., Firoozabadi A. (2003) Numerical simulation of water injection in fractured media using the discretefracture model and the Galerkin method, SPE Reserv. Eval. Eng. 6, 02, 117-126.

20 Ahmed R., Edwards M.G., Lamine S., Huisman B.A.H., Pal M. (2015) Control-volume distributed multi-point flux approximation coupled with a lower-dimensional fracture model, J. Comput. Phys. 284, 462-489. ISSN 0021-9991. https://doi.org/10.1016/jjcp.2014.12.047. http://www. sciencedirect.com/science/article/pii/S0021999114008705.

21 Brenner K., Hennicker J., Masson R., Samier P. (September 2016) Gradient discretization of hybrid-dimensional Darcy flow in fractured porous media with discontinuous pressures at matrix-fracture interfaces, IMA J. Numer. Anal. https://doi. org/10.1093/imanum/drw044. URL https://hal.archivesouvertes.fr/hal-01192740.

22 Brenner K., Hennicker J., Masson R., Samier P. (2018) Hybrid-dimensional modelling of two-phase flow through fractured porous media with enhanced matrix fracture transmission conditions, J. Comput. Phys. 357, 100-124. ISSN 0021-9991. https://doi.org/10.1016/j.jcp.2017.12.003. http://www.sciencedirect.com/science/article/pii/ S0021999117308781.

23 Brenner K., Groza M., Guichard C., Masson R. (2015) Vertex approximate gradient scheme for hybrid dimensional two-phase Darcy flows in fractured porous media, ESAIM: Math. Model. Numer. Anal. 49, 2, 303-330. https://doi.org/ $10.1051 / \mathrm{m} 2 \mathrm{an} / 2014034$.

24 Antonietti P.F., Formaggia L., Scotti A., Verani M., Verzotti N. (2016) Mimetic finite difference approximation of flows in fractured porous media, ESAIM: M2AN 50, 3, 809-832. https://doi.org/10.1051/m2an/2015087.

25 Scotti A., Formaggia L., Sottocasa F. (2017) Analysis of a mimetic finite difference approximation of flows in fractured porous media, ESAIM: M2AN. https://doi.org/10.1051/ m2an/2017028.

26 Benedetto M.F., Berrone S., Pieraccini S., Scialò S. (2014) The virtual element method for discrete fracture network simulations, Comput. Methods Appl. Mech. Eng. 280, 0, 135-156. ISSN 0045-7825. https://doi.org/10.1016/j.cma.2014.07.016.

27 Benedetto M.F., Berrone S., Borio A., Pieraccini S., Scialò S. (2016) A hybrid mortar virtual element method for discrete fracture network simulations, J. Comput. Phys. 306, 148-166. ISSN 0021-9991. https://doi.org/10.1016/jjcp. 2015.11.034. http://www.sciencedirect.com/science/article/ pii/S0021999115007743.

28 Fumagalli A., Keilegavlen E. (2018) Dual virtual element method for discrete fractures networks, SIAM J. Sci. Comput. 40, 1, B228-B258. https://doi.org/10.1137/ 16M1098231.

29 da Veiga L.B., Brezzi F., Cangiani A., Manzini G., Marini L.D., Russo A. (2013) Basic principles of virtual element methods, Math. Models Methods Appl. Sci. 23, 01, 199-214. https://doi.org/10.1142/S0218202512500492.

30 da Veiga L.B., Brezzi F., Marini L.D., Russo A. (2014) The hitchhiker's guide to the virtual element method, Math. Models Methods Appl. Sci. 24, 08, 1541-1573. https://doi. org/10.1142/S021820251440003X.

31 da Veiga L.B., Brezzi F., Marini L.D., Russo A. (Jun 2014) H (div) and $\mathrm{H}$ (curl)-conforming VEM, Numer. Math. 133, 2, 303-332. ISSN 0945-3245. https://doi.org/10.1007/s00211015-0746-1.

32 da Veiga L.B., Brezzi F., Marini L.D., Russo A. (2016) Mixed virtual element methods for general second order elliptic problems on polygonal meshes, ESAIM: M2AN 50, 3, 727-747. https://doi.org/10.1051/m2an/2015067.

33 Brezzi F., Falk R.S., Marini D.L. (2014) Basic principles of mixed virtual element methods, ESAIM: M2AN 48, 1227-1240. https://doi.org/10.1051/m2an/2013138.

34 Frih N., Martin V., Roberts J.E., Saâda A. (2012) Modeling fractures as interfaces with nonmatching grids, Comput. Geosci. 16, 4, 1043-1060. ISSN 1420-0597. https://doi.org/ $10.1007 / \mathrm{s} 10596-012-9302-6$.

35 Nordbotten J.M., Boon W., Fumagalli A., Keilegavlen E. (2018) Unified approach to discretization of flow in fractured porous media, Comput. Geosci. ISSN 1573-1499. https://doi. org /10.1007/s10596-018-9778-9.

36 Berrone S., Pieraccini S., Scialò S. (2013) On simulations of discrete fracture network flows with an optimization-based extended finite element method, SIAM J. Sci. Comput. 35, 2, 908-935. https://doi.org/10.1137/120882883. 
37 D'Angelo C., Scotti A. (2012) A mixed finite element method for Darcy flow in fractured porous media with nonmatching grids, Math. Model. Numer. Anal. 46, 02, 465-489. https://doi.org/10.1051/m2an/2011148.

38 Del Pra M., Fumagalli A., Scotti A. (2017) Well posedness of fully coupled fracture/bulk Darcy flow with XFEM, SIAM J. Numer. Anal. 55, 2, 785-811. https://doi.org/10.1137/ $15 \mathrm{M} 1022574$.

39 Fumagalli A., Scotti A. (2013) A numerical method for twophase flow in fractured porous media with non-matching grids, Adv. Water Res. 62, Part C(0), 454-464. ISSN 0309-1708. https://doi.org/10.1016/j.advwatres.2013.04.001. URL https://www.sciencedirect.com/science/article/pii/ S0309170813000523. Computational Methods in Geologic $\mathrm{CO}_{2}$ Sequestration.

40 Fumagalli A., Scotti A. (April 2014) An efficient XFEM approximation of Darcy flows in arbitrarily fractured porous media, Oil Gas Sci. Technol. - Rev. IFP Energies nouvelles 69, 4, 555-564. https://doi.org/10.2516/ogst/2013192. URL https://ogst.ifpenergiesnouvelles.fr/articles/ogst/abs/2014/ 04/ogst130007/ogst130007.html.

41 Tene M., Al Kobaisi M.S., Hajibeygi H. (2016) Multiscale projection-based embedded discrete fracture modeling approach (f-ams-pedfm), ECMOR XIV-15th European Conference on the Mathematics of Oil Recovery, August 29-1 September 2016, Beurs van Berlage, EAGE. https://doi.org/10.3997/2214-4609.201601890.

42 Fumagalli A., Zonca S., Formaggia L. (July 2017) Advances in computation of local problems for flow-based upscaling in fractured reservoirs, Math. Comput. Simul. 137, 299-324. https://doi.org/10.1016/j.matcom.2017.01.007. URL https:// www.sciencedirect.com/science/article/pii/S0378475417300320.

43 Li L., Lee S.H. (2008) Efficient field-scale simulation of black oil in a naturally fractured reservoir through discrete fracture networks and homogenized media, SPE Reserv. Eval. Eng. 11, 750-758. https://doi.org/10.2118/103901-PA.

44 Berrone S., Pieraccini S., Scialò S. (2013) A PDE-constrained optimization formulation for discrete fracture network flows, SIAM J. Sci. Comput., 35, 2, B487-B510. https://doi.org/ $10.1137 / 120865884$.

45 Formaggia L., Fumagalli A., Scotti A., Ruffo P. (2014) A reduced model for Darcy's problem in networks of fractures, ESAIM: Math. Model. Numer. Anal. 48, 1089-1116. https://doi.org/10.1051/m2an/2013132. URL https://www. esaim-m2an.org/articles/m2an/abs/2014/04/m2an130132/ m2an130132.html.
46 Boon W.M., Nordbotten J.M., Vatne J.E. (2017) Mixeddimensional elliptic partial differential equations. Technical report, arXiv:1710.00556 [math.AP].

47 Fumagalli A., Scotti A. (2013) A reduced model for flow and transport in fractured porous media with nonmatching grids, in: Cangiani A., Davidchack R.L., Georgoulis E., Gorban A.N., Levesley J., Tretyakov M.V. (eds), Numerical mathematics and advanced applications 2011, Berlin, Heidelberg, Springer, pp. 499-507. ISBN 978-3-642-33133-6. https://doi. org/10.1007/978-3-642-33134-3 53.

48 Grisvard P. (1985) Elliptic problems in non-smooth domains, Vol. 24, Monographs and studies in mathematics, Pitman.

49 Geuzaine C., Remacle J.-F. (2009) Gmsh: A 3-d finite element mesh generator with built-in pre- and post-processing facilities, Int. J. Numer. Methods Eng. 79, 11, 1309-1331. ISSN 1097-0207. https://doi.org/10.1002/nme.2579.

50 Fumagalli A. (Dec. 2018) Dual virtual element method in presence of an inclusion, Appl. Math. Lett. 86, 22-29. https://doi.org/10.1016/j.aml.2018.06.004. URL https://www. sciencedirect.com/science/article/pii/S0893965918301812.

51 Flemisch B., Berre I., Boon W., Fumagalli A., Schwenck N., Scotti A., Stefansson I., Tatomir A. (January 2018) Benchmarks for single-phase flow in fractured porous media, $A d v$. Water Res. 111, 239-258. https://doi.org/10.1016/j.advwatres.2017.10.036. URL https://www.sciencedirect.com/science/ article/pii/S0309170817300143.

52 Keilegavlen E., Fumagalli A., Berge R., Stefansson I., Berre I. (2017) Porepy: An open source simulation tool for flow and transport in deformable fractured rocks. Technical report, arXiv: 1712.00460 [cs.CE]. URL https://arxiv.org/abs/1712.00460.

53 Flemisch B., Rainer H. (2008) Numerical investigation of a mimetic finite difference method, in: Finite volumes for complex applications $V$ - Problems and perspectives, Wiley-VCH, Germany, pp. 815-824.

54 Geiger S., Dentz M., Neuweiler I. (2011) A novel multi-rate dual-porosity model for improved simulation of fractured and multiporosity reservoirs, SPE Reservoir Characterisation and Simulation Conference and Exhibition, 9-11 October, Abu Dhabi.

55 Sausse J., Dezayes C., Dorbath L., Genter A., Place J. (2010) $3 \mathrm{~d}$ model of fracture zones at soultzsous-for Sts based on geological data, image logs, induced microseismicity and vertical seismic profiles, C. R. Geosci. 342, 7, 531-545. ISSN 1631-0713. doi: http://doi.org/10.1016/j.crte.2010.01. 011. URL http://www.sciencedirect.com/science/article/pii/ S1631071310000489. 